\title{
Nanovibrational Stimulation of Mesenchymal Stem Cells Induces Therapeutic Reactive Oxygen Species and Inflammation for Three- Dimensional Bone Tissue Engineering
}

\author{
Wich Orapiriyakul, Monica P. Tsimbouri, Peter Childs, Paul Campsie, Julia Wells, \\ Marc A. Fernandez-Yague, Karl Burgess, K. Elizabeth Tanner, Manlio Tassieri, Dominic Meek, \\ Massimo Vassalli, Manus J. P. Biggs, Manuel Salmeron-Sanchez, Richard O. C. Oreffo, Stuart Reid, \\ and Matthew J. Dalby*
}

Cite This: https://dx.doi.org/10.1021/acsnano.0c03130

Read Online

ACCESS | Llll Metrics \& More | 回 Article Recommendations

SI Supporting Information

ABSTRACT: There is a pressing clinical need to develop cellbased bone therapies due to a lack of viable, autologous bone grafts and a growing demand for bone grafts in musculoskeletal surgery. Such therapies can be tissue engineered and cellular, such as osteoblasts, combined with a material scaffold. Because mesenchymal stem cells (MSCs) are both available and fast growing compared to mature osteoblasts, therapies that utilize these progenitor cells are particularly promising. We have developed a nanovibrational bioreactor that can convert MSCs into bone-forming osteoblasts in two- and three-dimensional, but the mechanisms involved in this osteoinduction process remain unclear. Here, to elucidate this mechanism, we use increasing vibrational amplitude, from $30 \mathrm{~nm}(\mathrm{~N} 30)$ to $90 \mathrm{~nm}$
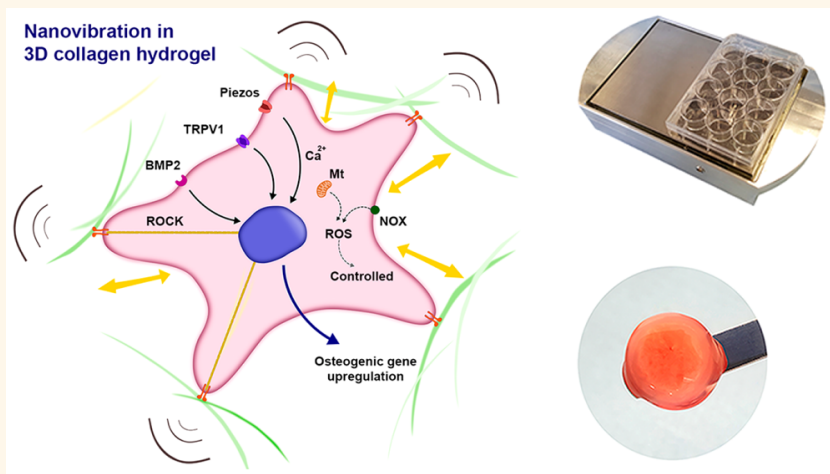
(N90) amplitudes at $1000 \mathrm{~Hz}$ and assess MSC metabolite, gene, and protein changes. These approaches reveal that dosedependent changes occur in MSCs' responses to increased vibrational amplitude, particularly in adhesion and mechanosensitive ion channel expression and that energetic metabolic pathways are activated, leading to low-level reactive oxygen species (ROS) production and to low-level inflammation as well as to ROS- and inflammation-balancing pathways. These events are analogous to those that occur in the natural bone-healing processes. We have also developed a tissue engineered MSC-laden scaffold designed using cells' mechanical memory, driven by the stronger N90 stimulation. These mechanistic insights and cell-scaffold design are underpinned by a process that is free of inductive chemicals.

KEYWORDS: mesenchymal stem cells, nanovibration, mechanotransduction, bioreactor, bone tissue engineering

$\mathrm{B}$ one is the second-most grafted tissue after blood in humans and is used in a wide range of musculoskeletal surgeries. ${ }^{1-3}$ However, the use of autografts from patient donor sites is limited and has a high incidence of morbidity. ${ }^{1-3}$ Allograft is therefore widely used but also suffers from a lack of donor material and is acellular and so lacks biological activity. ${ }^{1-3}$ Cellular therapies, used in combination with scaffolding materials, represent a future source of bone graft and regeneration approaches. As such, a growing number of mesenchymal stem cell (MSC)-based therapies are in clinical trial. ${ }^{4}$ We note that the term MSC is widely used and often refers to adherent stromal cells, as is typically the case in cell therapies. ${ }^{5}$ However, here we use the term MSC to more accurately refer to skeletal stem cells selected from bone marrow stroma using Stro1. ${ }^{6,7}$ These are a clonogenic population of nonhematopoietic bone marrow stromal cells that can recreate cartilage, bone, adipocytes, and hematopoiesis-supporting stroma. ${ }^{6,7}$

Received: April 14, 2020

Accepted: July 13, 2020

Published: July 13, 2020 
A

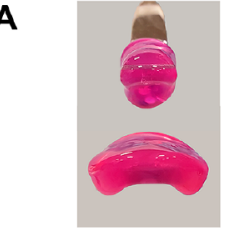

B

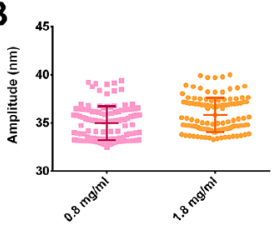

$\mathbf{F}$

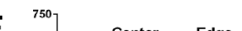

F $\left.{ }_{700}^{750}\right] \quad$ - Center - Edge

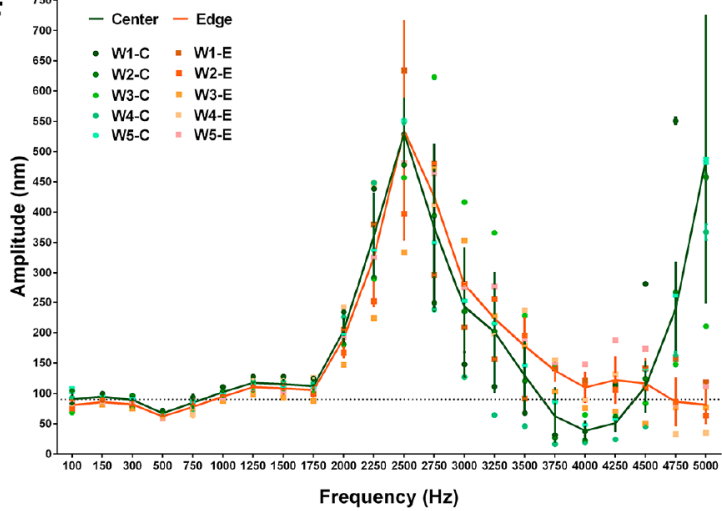

G

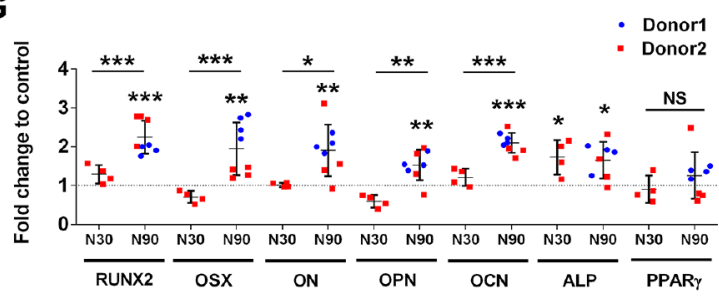

C

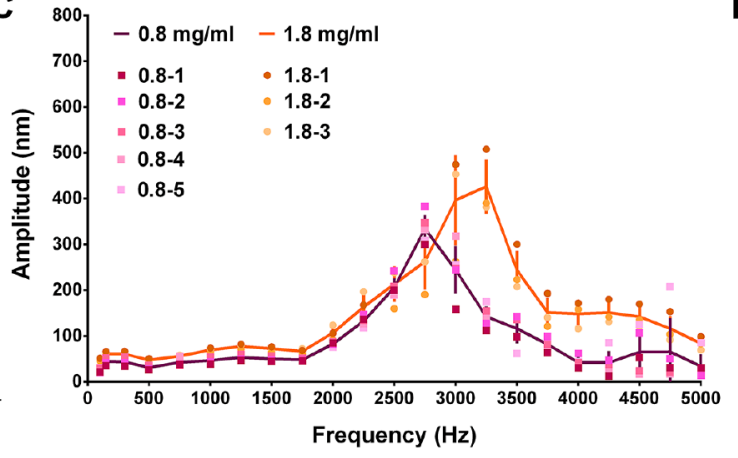

D

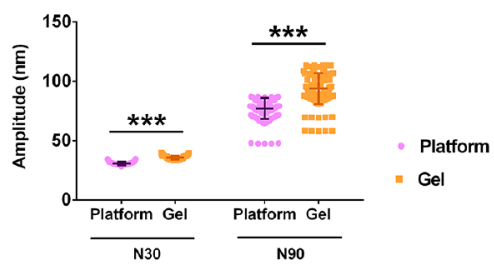

E

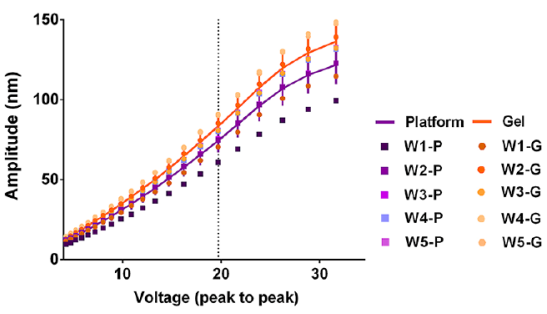

H

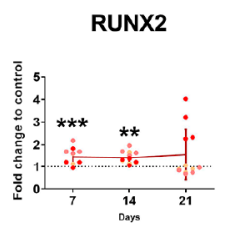

ALP

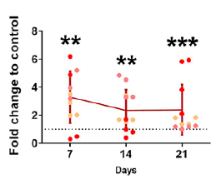

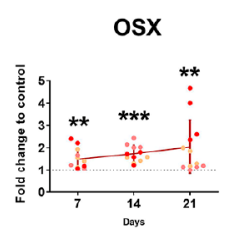

OPN

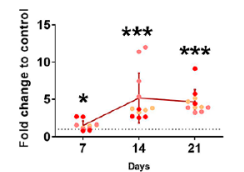

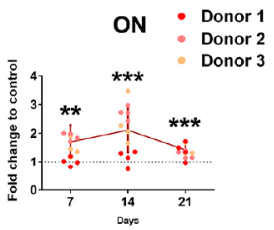

OCN

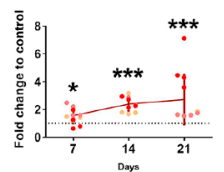

I

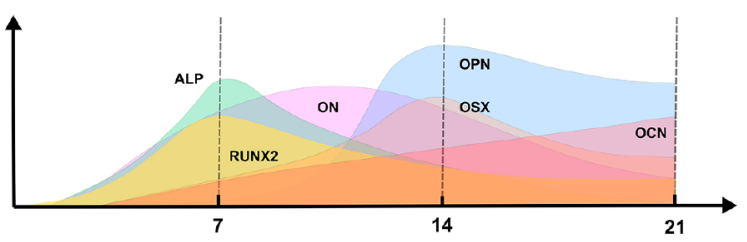

Figure 1. 3D MSC osteogenesis with N30 and N90 nanostimulation. (A) Collagen gel constructed with $0.8 \mathrm{mg} / \mathrm{mL}$ (top) and $1.8 \mathrm{mg} / \mathrm{mL}$ (bottom) (note that both are front views, and the gel diameter was $13 \mathrm{~mm}$ before removing from the well). (B) Interferometry showing that 0.8 and $1.8 \mathrm{mg} / \mathrm{mL}$ collagen gels vibrate as expected with $\mathrm{N} 30$ stimulation $(n=24)$. (C) Using interferometry, resonance effects were seen at frequencies $>2000 \mathrm{~Hz}$, but no resonance peaks were seen at $1000 \mathrm{~Hz}$, for both 0.8 and $1.8 \mathrm{mg} / \mathrm{mL}$ collagen gels $(n=3-5)$. (D) Interferometry results for $\mathrm{N} 30$ and $\mathrm{N} 90$ nanostimulation, showing good fidelity of vibration in the $1.8 \mathrm{mg} / \mathrm{mL}$ collagen gel $(n=24)$. (E) Interferometry showed a linear voltage-amplitude relationship for both the vibration platform and the $1.8 \mathrm{mg} / \mathrm{mL}$ collagen gel $(n=5)$ between 12 and $27 \mathrm{Vpp}$. (F) No resonance frequencies were seen at $1000 \mathrm{~Hz}$ at either the center or edge of the $1.8 \mathrm{mg} / \mathrm{mL}$ collagen gels $(n=5)$. (G) Osteogenic marker gene expression in MSCs, as assessed by qPCR for N90 compared to N30 (or control), after 9 days of culture in 3D nanovibrational stimulation conditions in $1.8 \mathrm{mg} / \mathrm{mL}$ collagen gels. Osteogenic marker gene expression was enhanced in N90 conditions, compared to N30 or control conditions $(d=2, r=4, t=3)$. (H) Osteogenic transcript expression in $\mathrm{N} 90$ conditions in $1.8 \mathrm{mg} / \mathrm{mL}$ collagen gels at days 7 , 14, and 21 of culture. (I) Schematic of expression maxima with time $(d=3, r=4, t=3)$. Significance calculated using ANOVA with Tukey multiple comparison, where $*=p<0.05, * *=p<0.01$, and $* * *=p<0.001$. Error bars represent means \pm SD. The data shows good fidelity and improved efficacy of 3D nanostimulation with change in gel stiffness and with increased vibration amplitude. Abbreviations: $d=$ number of donors assessed; $r=$ number of wells tested; and $t=$ technical replicates. Donors are MSCs derived from different donor sources.

We have previously reported the development of a nanovibrational bioreactor that can stimulate MSC differentiation toward osteogenesis in two-dimensional $(2 \mathrm{D})^{8}$ or threedimensional $(3 \mathrm{D})^{9}$ without the use of defined media, chemicals, or highly specialized equipment (see supplementary Figure 1 for an image of the bioreactor). This approach offers significant advantages because it enables media, standard consumables, and MSC banks that have already been approved for use to be utilized. For example, in this study we used 6- and 24-well plates with our nanovibrational bioreactor by simply attaching them to a vibrating top-plate with magnets. The use of standard consumables also enables cells to be cultured in wells with scaffold materials, such as hydrogels, enabling us to think beyond just cell manufacture and toward tissue engineering.
The bioreactor employs the reverse piezo effect, in which a voltage is used to cause a mechanical expansion of a material, in this case a piezo active ceramic. The piezo ceramics are sandwiched between a large mass (aluminum block) and the ferrous top plate (which provides a magnetic interface). ${ }^{10} \mathrm{We}$ have previously trialled a range of frequencies using this set up, with a fixed amplitude of $30 \mathrm{~nm}$, and have found $1000 \mathrm{~Hz}$ to be optimal for MSC osteoinduction. ${ }^{8}$

Despite our ability to induce osteogenesis using this approach, we know little about how mechanistically nanovibrational mechanotransduction in MSCs induces osteogenic differentiation. We have previously reported altered cell adhesion and RhoA kinase (ROCK) activity in MSCs cultured in 2D in the nanovibrational bioreactor, ${ }^{8}$ and we have also previously 
A

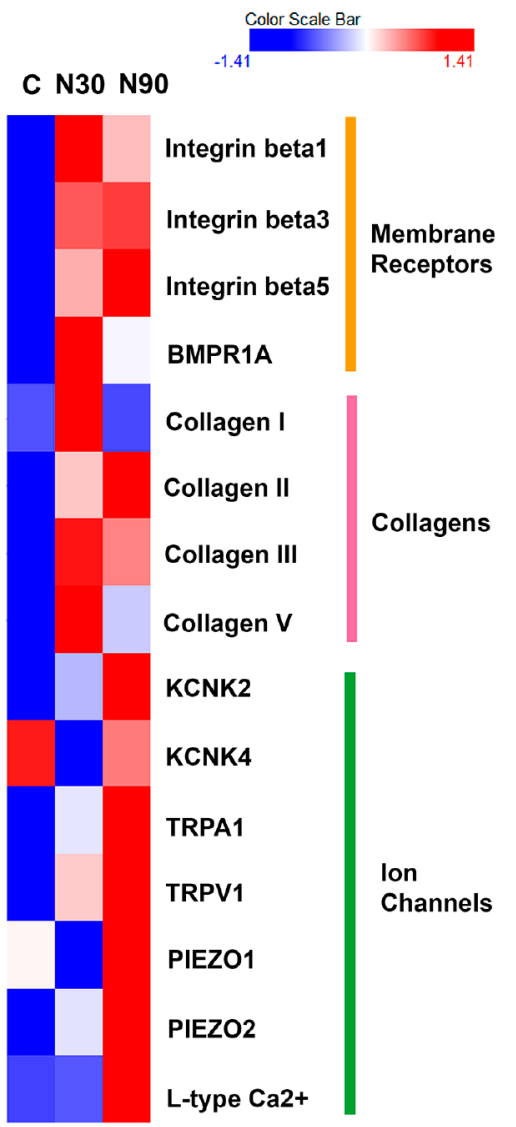

B

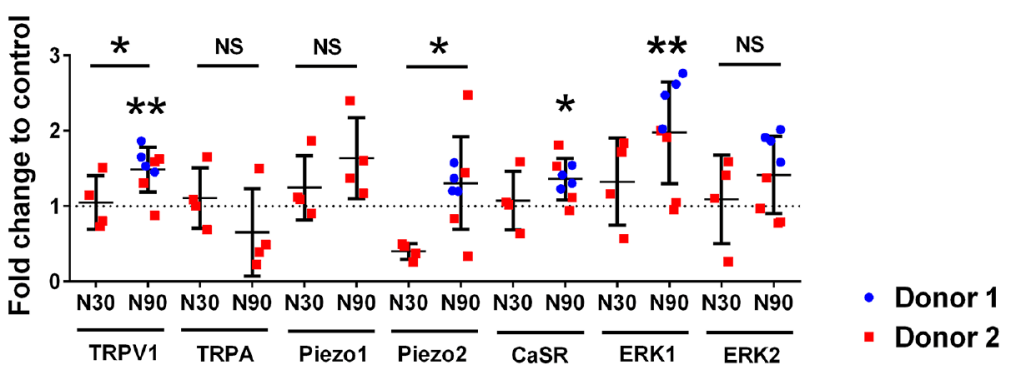

C
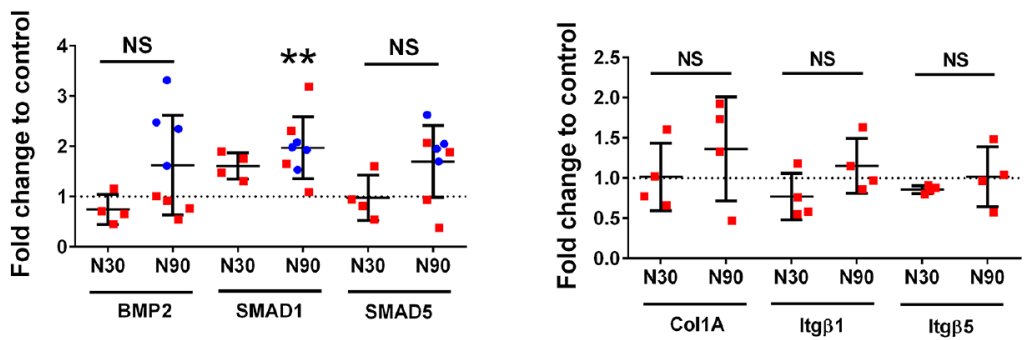

D

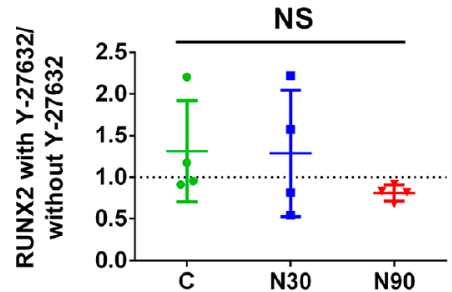

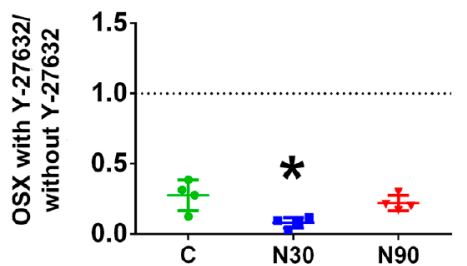

Figure 2. Adhesion and ion channel expression by MSCs when cultured with vibrational amplitude of N30 or N90. (A) Protein array data, presented as a heatmap. It shows the expression levels of a range of adhesion, extracellular, and ion channel proteins in MSCs cultured in control conditions (C) and in conditions of N30 and N90 vibrational amplitude, after 9 days of stimulation $(d=2, r=4, t=3)$. Red denotes increased expression, and blue denotes reduced expression, relative to control. (B, C) Ion channel expression (B), BMP family member's expression (C, left), and adhesion molecule expression (C, right), as assessed by qPCR, for control, N30 and N90 cultures after 9 days of stimulation $(d=2, r=$ 4, $t=3$ ). (D) qPCR analysis of RUNX2 and OSX expression, with or without ROCK inhibition (Y-27632), for control, N30 and N90 cultures after 9 days of stimulation $(d=1, r=4, t=3)$. Significance calculated using ANOVA with Tukey multiple comparison where $*=p<0.05$ and $* *=$ $p<0.01$. Error bars represent means \pm SD. Data show that ion channel and extracellular matrix and adhesion proteins are more highly expressed in $\mathrm{N} 90$ conditions relative to $\mathrm{N} 30$ conditions and that inhibiting ROCK has only a small effect on osteogenesis. Abbreviations, $d=$ number of donors assessed; $r=$ number of wells tested; and $t=$ technical replicates.

identified TRPV1, transient receptor potential cation channel subfamily $\mathrm{V}$ member 1 , as being implicated in $3 \mathrm{D}$ nanovibrational osteogenesis.

We hypothesize that increasing nanovibrational amplitude will enhance an osteogenic effect and related cell signaling. From our findings, we propose that increasing the nanovibrational amplitude that MSCs are exposed to exaggerates the underlying osteogenic mechanism, allowing us to further dissect MSC mechanotransduction mechanisms induced by nanovibration.

\section{RESULTS AND DISCUSSION}

Optimization of 3D Nanovibrational Cultures. Type I collagen is a widely used 3D hydrogel scaffold, which we have previously used in nanovibrational experiments at $0.8 \mathrm{mg} / \mathrm{mL}$ concentration. ${ }^{9}$ An advantage of this collagen formulation is its low stiffness $(E=\sim 26 \mathrm{~Pa}$ at $0.8 \mathrm{mg} / \mathrm{mL}$, supplementary Figure $2 \mathrm{~A}$ ), which is well below the $30-40 \mathrm{kPa}$ stiffness required to drive MSC osteogenesis ${ }^{11,12}$ and its good biocompatibility as shown using alamar blue (supplementary Figure 2C; please note that live-dead staining showing no reduction in cell viability for $\mathrm{N} 30$ is available in ref 9). Importantly for nanovibrational stimulation, it adheres to the sides of cell culture plates, thereby providing mechanical integration with the plate. As a hydrogel, it is incompressible. ${ }^{13}$ It thus acts as a solid volume when vibrated in a contained environment, such as the wells of a culture plate, providing good vibration propagation throughout its volume 9 (Figure 1B). However, the action of cells within the collagen gel can induce the gel to contract from the edge of the well over long-term osteogenic cultures, which typically take 28 days to reach mineralization. ${ }^{14,15}$

In order to overcome this issue, we trialled a $1.8 \mathrm{mg} / \mathrm{mL}$ collagen gel. While this gel formulation is stiffer $(E=\sim 161 \mathrm{~Pa}$, supplementary Figure 2A), it remains significantly below the stiffness required to induce MSC osteogenesis $(>20 \mathrm{kPa}) \cdot{ }^{11,12}$ It was also easier to handle (as shown in Figure 1A), which could have positive implications in the operating theater, where surgeons need to remove a cell product from a dish to place into a patient. Importantly, vibrational fidelity was similar to that of the $0.8 \mathrm{mg} / \mathrm{mL}$ gel, with a $30 \mathrm{~nm}$ displacement at the vibration plate surface inducing $35 \mathrm{~nm}$ vibrations in both the 0.8 and 1.8 $\mathrm{mg} / \mathrm{mL}$ gels, as indicated by laser interferometry (Figure 1B). Furthermore, little evidence of resonance effect was seen in any 

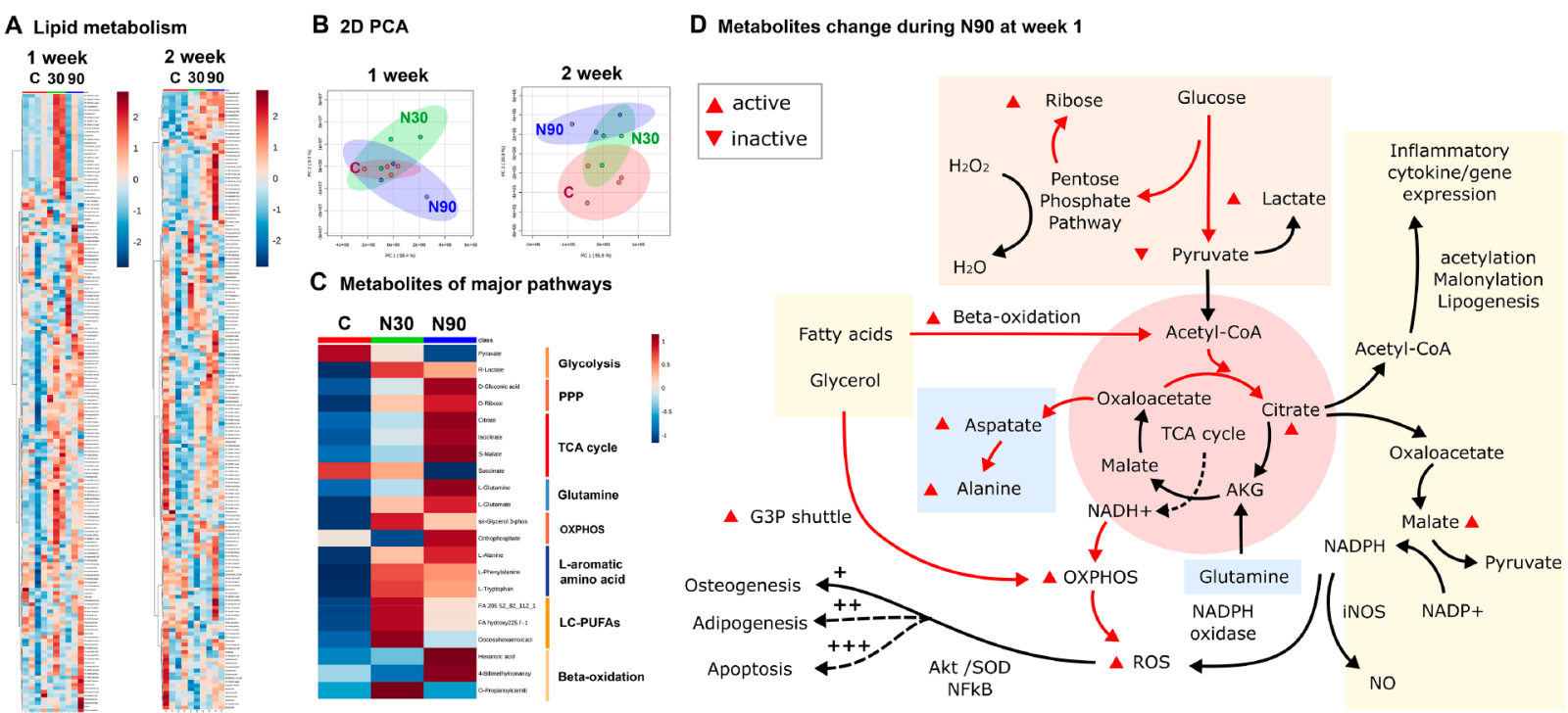

Figure 3. Untargeted metabolomic analysis of MSCs cultured in N30 and N90 stimulation conditions. (A) Lipid heatmaps of MSCs after 1 and 2 weeks of nanostimulation at N30 and N90 amplitudes. (B) PCA of MSC lipid data after 1 and 2 weeks of culture in N30 and N90 nanostimulation conditions, compared to control. (C) Observed metabolite changes in ROS pathways following 1 week of culture under N30 or N90 conditions. (D) Schematic of potential pathways derived from the heatmap data $(d=1, r=4, t=1)$. The data indicate the activation of ROS and redox-balancing pathway occurs in MSCs cultured in conditions of increasing nanostimulation amplitude. Abbreviations: $d=$ number of donors assessed; $r=$ number of wells tested; and $t=$ technical replicates.

replicates of the 0.8 and $1.8 \mathrm{mg} / \mathrm{mL}$ gels at $1000 \mathrm{~Hz}$ driving frequency (Figure 1C). By looking at collagen-plastic detachment over a longer culture period, we found that while the 0.8 $\mathrm{mg} / \mathrm{mL}$ gels contracted within a 30 day culture period, with typical cell seeding of $40,000 \mathrm{MSCs} / \mathrm{mL}$ collagen (supplementary Figure $2 \mathrm{~B}$ ), the $1.8 \mathrm{mg} / \mathrm{mL}$ gels did not contract until $>70$ days when seeded with either 40,000 or 80,000 cells $/ \mathrm{mL}$ and exposed to $1000 \mathrm{~Hz}, 30 \mathrm{~nm}$ vibrational stimulation. This time period extends way beyond the usual duration of our MSC nanovibration experiments (supplementary Figure 2B).

A key aim of this research was to investigate if a larger amplitude can induce more pronounced changes to allow us to infer cell mechanism with more clarity. To investigate this, we selected a second amplitude of $90 \mathrm{~nm}$, which we used in addition to a previously tested amplitude of $30 \mathrm{~nm}$ (referred to as N30 and N90, respectively). For both gels, we observed a slight increase in amplitude, relative to that of the top plate, from 30 to $\sim 35 \mathrm{~nm}$ with $\mathrm{N} 30$ and from 90 to $\sim 100 \mathrm{~nm}$ with N90 (Figure 1D). We note that the voltage-amplitude relationship was linear between 12 and $27 \mathrm{Vpp}$ (voltage peak-to-peak, the region in which we operate) in the $1.8 \mathrm{mg} / \mathrm{mL}$ collagen gel, as it was when measured on the bioreactor top plate (Figure 1E), again demonstrating the fidelity of the system.

We next assessed the resonance using interferometry. At 1000 $\mathrm{Hz}$, the bioreactor generated reliable displacements without resonance problems at the edge and the center of the $1.8 \mathrm{mg} / \mathrm{mL}$ hydrogels (Figure $1 \mathrm{~F}$ ). Osteogenic marker expression was also assessed by qPCR. After 9 days of MSC stimulation in 3D, using $1.8 \mathrm{mg} / \mathrm{mL}$ gels and $\mathrm{N} 30$ stimulation, alkaline phosphatase (ALP) expression was detected (Figure 1G). However, osteogenic stimulation was significantly more pronounced following N90 stimulation, with several osteoblast markers expressed after 9 days, including ALP, runt related transcription factor 2 (RUNX2), osterix (OSX), osteonectin (ON), osteopontin (OPN), and osteocalcin (OCN) (Figure 1G). Peroxisome proliferator activated receptor $\gamma$ (PPAR $\gamma)$ expression, an adipocyte marker, was also assessed to gauge whether the nanovibrational effect was osteospecific. No induction of PPAR $\gamma$ was observed with N30 or N90 stimulation at 9 days of culture (Figure $1 \mathrm{G}$ ). Alamar blue staining showed that there were no cytotoxic effects of N30 or N90 stimulation in the $1.8 \mathrm{mg} / \mathrm{mL}$ collagen gels or in the control conditions with/ without osteogenic media (supplementary Figure 2C).

Higher Amplitude Stimulation Increases Osteogenesis and Ion Channel Expression. We next assessed how nanostimulation at N30 and N90 conditions affected the expression of adhesions and ion channels that have been previously implicated in N30-stimulated MSC osteogenesis. ${ }^{9}$ To do so, we used a protein array containing a range of receptors and channels. Using this array, we observed the upregulated expression of $\beta 1,3$, and 5 integrins after 9 days of MSC culture in both N30 and N90 conditions (Figure 2A). These integrins function as receptors for a wide range of extracellular matrix (ECM) proteins. A range of collagens were also upregulated in N30- and N90-stimulated MSCs (Figure 2A); we note that integrin $\beta 1$ is used by cells to attach to collagens. ${ }^{16}$ Bone morphogenetic protein 2 (BMP2) receptor BMPR1 was also upregulated following nanovibrational stimulation (Figure 2A). Most interestingly, however, was the pattern of ion channel expression by MSCs. In agreement with previous results, ${ }^{9} \mathrm{~N} 30$ conditions stimulated the expression of transient receptor potential cation channel subfamily $\mathrm{V}$ member 1 (TRPV1) (Figure 2A). However, under N90 conditions, TRPV1 was more highly expressed, as were TRPA1, Piezol and 2, and potassium channel subfamily K member 2 (KCNK2) (Figure 2A), each of which are mechanosensitive ion channels that can be opened by stretch, for example, by membrane deformation ${ }^{17,18}$ or by myosin contracting the cytoskeleton. ${ }^{19,20}$ They also reportedly transduce high-frequency vibrational forces, such as in the ear $^{21,22}$ (we note that $1000 \mathrm{~Hz}$ is in the audible range). Moreover, KCNK2 (also known as TREK1) has been associated with low-frequency osteoinduction of MSCs via magnetic twisting cytometry. ${ }^{23}$ 
A

B
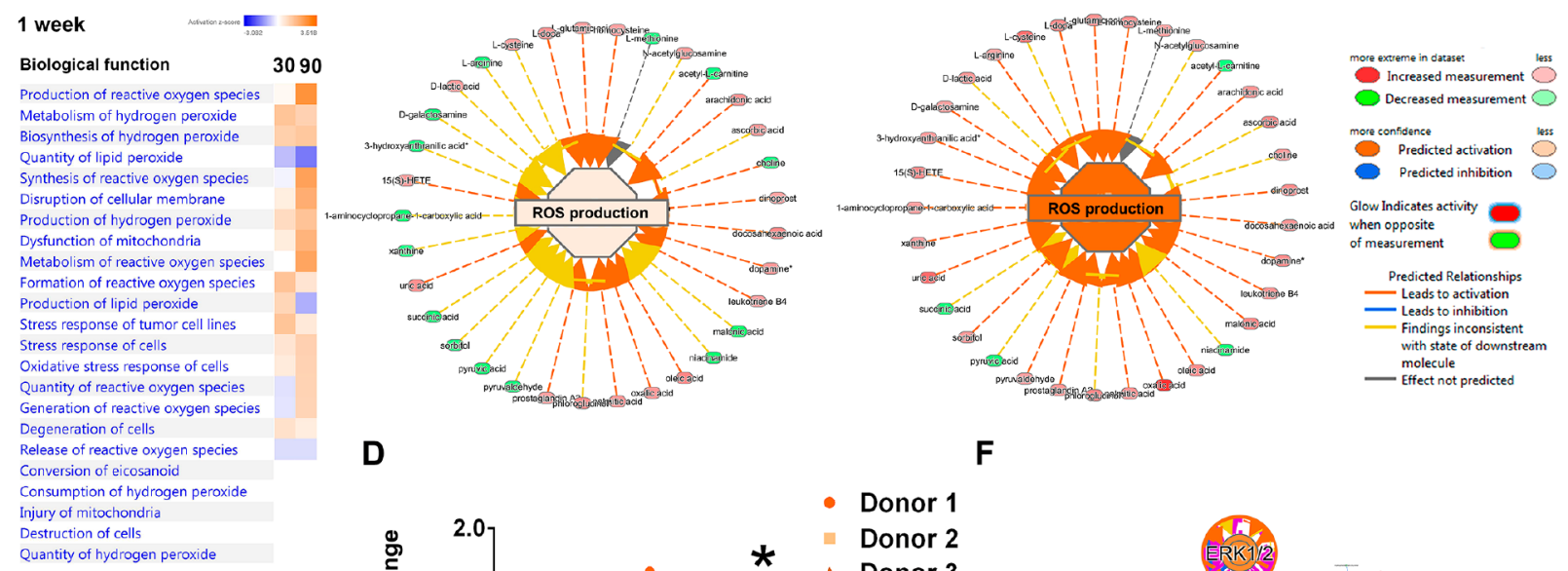

D

F
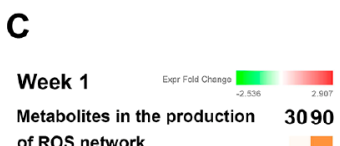
of ROS network

oxalic acid

uric acid
oleic acid

oleic acid
L-cysteine

phloroglucinol

docosahexaenoic acid

L-dopa

ascorbic acid

palmitic acid

15(S)-HETE

dopamine
arachidonic acid

dinoprost

L-homocysteine
leukotriene B4

leukotriene B4

L-glutamic acid
D-lactic acid

D-lactic acid
prostaglandin A2

$\mathrm{N}$-acetylglucosamin

$\mathrm{N}$-acetylglucosa
malonic acid

malonic aci
sorbitol

choline

3-hydroxyanthranilic acid

kanthine

pyruvaldehyde

L-methionine

D-galactos

-arginine

aminocyclopropane-1 carboxylic acid

acetyl-L-carnitine
pyruvic acid

succinic acid

succinic acid
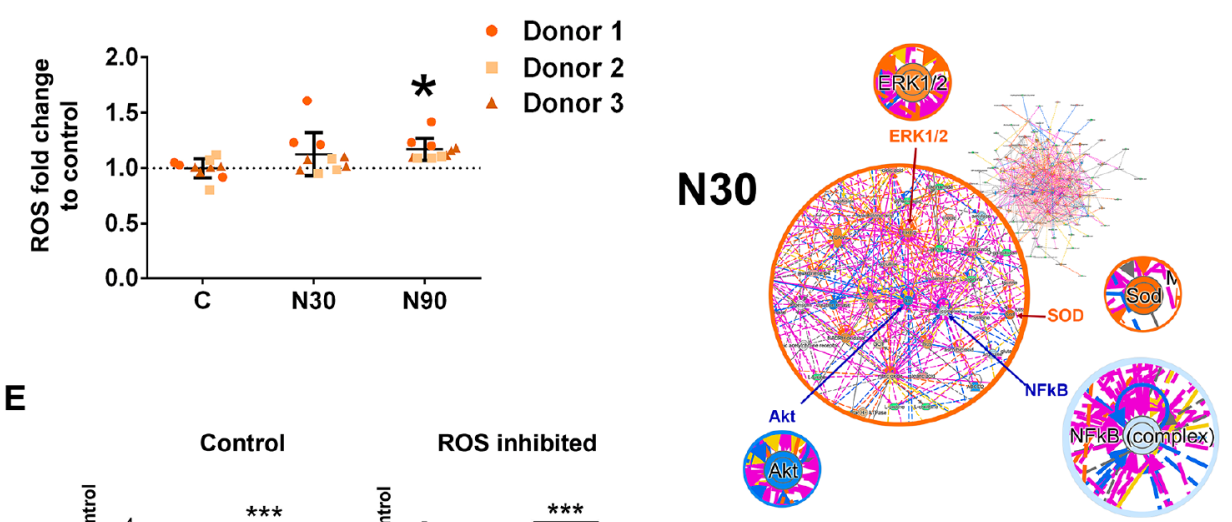

E
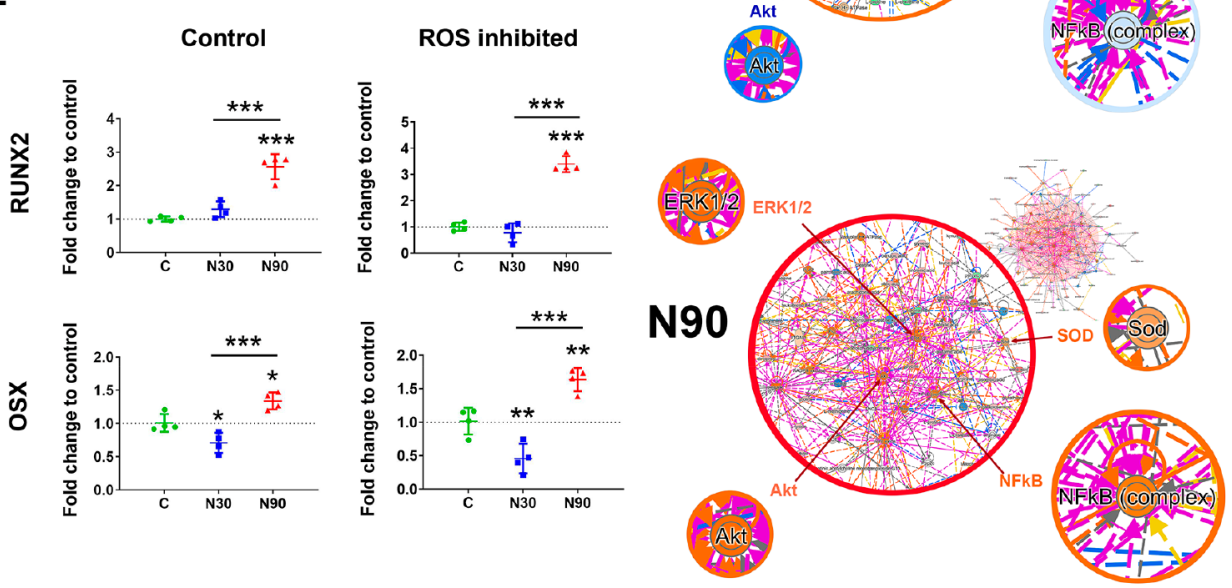

Figure 4. Metabolomic analysis of MSCs cultured with N30 and N90 nanostimulation. (A) Observation of ROS-based pathways. (B, C) Metabolites involved in ROS after MSCs were cultured for 7 days of under control, N30 and N90 conditions $(d=1, r=4, t=1)$. (D) DCF-DA flow analysis, showing that ROS levels increase with nanostimulation amplitude, reaching significance following MSC stimulation at N90 $(d=3$, $r=3, t=1$ ). (E) qPCR analysis of RUNX2 and $O S X$ expression in MSCs after 9 days of culture under N30 or N90 conditions. Both markers were upregulated following N90 stimulation in this donor cell line, with ROS inhibition having little effect on their expression $(d=1, r=4, t=3)$. (F) (top) Ingenuity pathway analysis of metabolite networks induced by N30 stimulation, showing the predicted upregulation of ERK1/2 (osteogenic commitment) and SOD (ROS) and the predicted repression of Akt and NFKB (REDOX balancing). (bottom) N90 stimulation is predicted to result in the activation of all these pathways $(d=1, \mathrm{r}=4, t=1)$. For (D and E), error bars represent means \pm SD. Significance calculated using ANOVA with Tukey multiple comparisons, where $*=p<0.05, * *=p<0.01$, and $* * *=p<0.001$. The data indicate that MSCs generate ROS while committing to osteogenesis and that, when the signal becomes stronger, the cells also activate REDOX balancing pathways. They also indicate that ROS itself is not a driver of osteogenesis. Abbreviations: $d=$ number of donors assessed; $r=$ number of wells tested; and $t$ $=$ technical replicates.

It is notable that Piezo1 is down-regulated in N30 conditions and upregulated in $\mathrm{N} 90$ conditions, compared to control. This might be a time-dependent phenomenon or a gated phenomenon, wherein higher levels of stimuli are more likely to activate threshold-dependent mechanisms. Indeed, the force-dependent activation of Piezol has been compared to a switch; ${ }^{24}$ for example, Piezo1 has been linked to ATP signaling in MSCs in a threshold-dependent manner. ${ }^{25}$

We assessed protein levels with the protein array and transcript levels with $\mathrm{qPCR}$ at the same time points. Ion channels showed less change at the transcript level than at the protein level, with N90 stimulation producing the greatest upregulation of TRPV1, Piezo2 and calcium sensing receptor (CaSR) expression, and of the downstream target, extracellular signal related kinase 1 (ERK1). Among the assessed BMP signaling family members, only the expression of small mothers against decapentaplegic 1 (SMAD1) was induced by N90 stimulation (Figure 2C, left), while the assessed collagen genes were not expressed by MSCs cultured with N30 or N90 stimulation (Figure 2C, right). Inhibiting cytoskeletal tension 
A

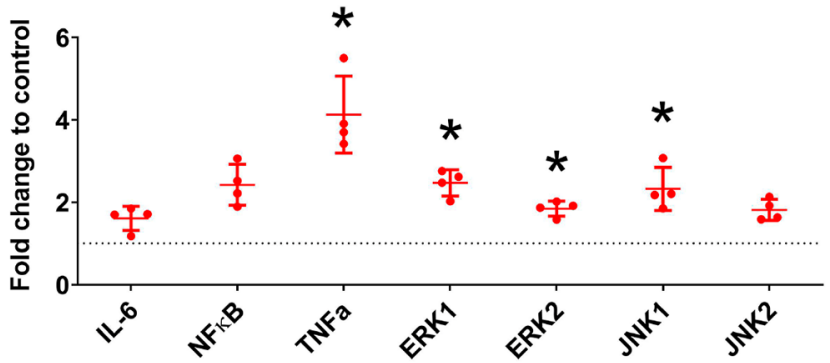

B
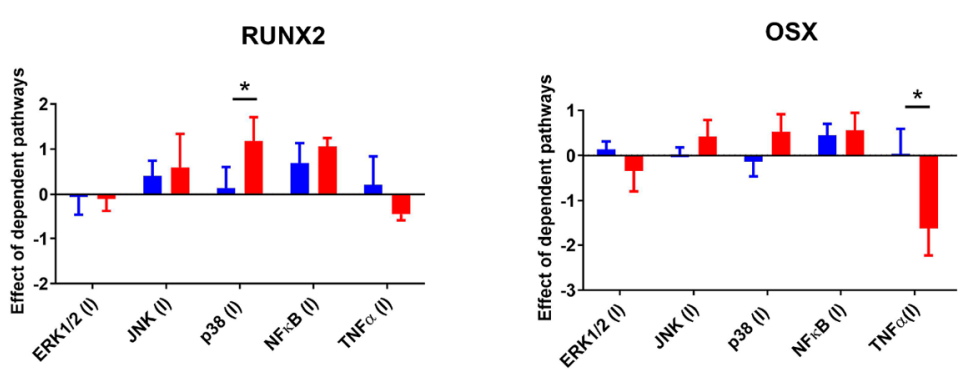

C

IL-6

NFkB
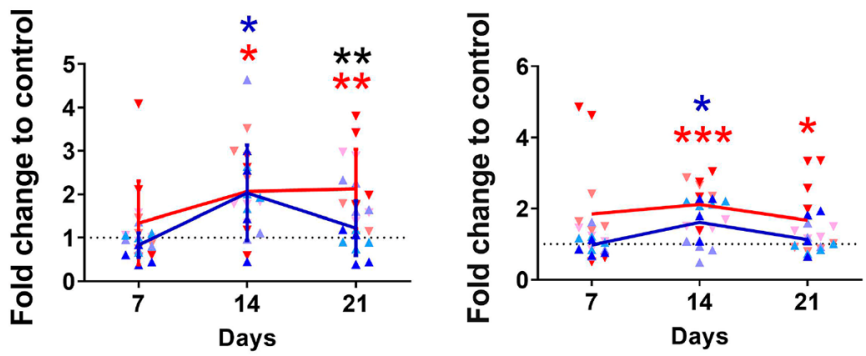

- N30 - Donor 1

4 N30 - Donor 2

4 N30 - Donor 3

- N90 - Donor 1

V N90 - Donor 2

N90 - Donor 3

$-\mathrm{N} 30$

- N90

D

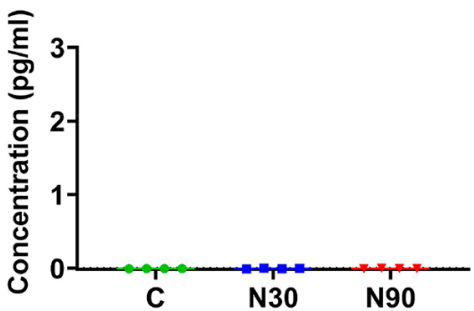

- C

- N30

- N90

Figure 5. Nanostimulation at N90 upregulates inflammatory markers in MSCs. (A) Increased expression of TNF $\alpha$, ERK1/2, and JNK1 in MSCs cultured with N90 stimulation for 9 days, as assessed by qPCR $(d=1, r=4, t=3)$. (B) Inhibition studies with qPRC for RUNX2 and osterix (OSX) showed that p38 MAPK inhibited RUNX2 expression and TNF $\alpha$ enhanced OSX expression with N90 stimulation compared to N30 $(d=$ $1, r=4, t=3)$. (C) IL-6 and NF $\kappa$ B show increased expression to day 14 of culture and decreased expression to day 21, as assessed by qPCR; increased expression was more persistent for N90 stimulation $(d=3, r=4, t=3)$. (D) IL-1 showed no detectable expression with either N30 or N90 stimulation, as assessed by ELISA $(d=1, r=4, t=1)$. Error bars represent means \pm SD, significance calculated using ANOVA with Tukey multiple comparison, where $*=p<0.05, * *=p<0.01$, and $* * *=p<0.001$ (note that blue and red asterisks show the significant difference of N30 and N90 to control, while black asterisks represent significant difference between N30 and N90). The data show a very low-level inflammatory response that is not detectable at the protein level. Abbreviations: $d=$ number of donors assessed; $r=$ number of wells tested; and $t$ $=$ technical replicates.

with the ROCK inhibitor, Y-27632, produced only a subtle loss of osteogenesis, with only $\mathrm{N} 30$ conditions indicating that intracellular tension is important for cell responses to $3 \mathrm{D}$ nanovibrational stimulation (Figure 2D).

From these results, we propose that vibrational amplitude at N90 provides a more powerful osteogenic cue than does N30 and that ion channel expression is particularly increased by this higher amplitude.

Reactive Oxygen Species and Nanovibrational Stimulation. To investigate further the cellular pathways that are altered by nanovibrational stimulation in MSCs, we took an untargeted metabolomics approach. Cells were lysed after culture for 1 or 2 weeks in control, N30 or N90 conditions, and then analyzed by liquid chromatography (LC)-orbitrap mass spectrometry (MS). ${ }^{26}$ Heatmap analysis and principle component analysis (PCA) revealed that lipids are the largest differentially regulated metabolite group (Figure 3A) and that the metabolome of N30 and N90 cultured cells MSCs diverged from each other and the control group (Figure 3B).

After 1 week of culture, the metabolites of major respirationrelated pathways, including glycolysis, the pentose phosphate pathway, TCA cycle, oxidative phosphorylation (OXPHOS), Laromatic amino acid, long-chain polyunsaturated fatty acids (LC-PUFAs), and $\beta$-oxidation were typically upregulated to 
differing levels by N30 and N90 conditions (Figure 3C). N90 conditions produced the most differentially regulated responses relative to unstimulated control conditions. The responses to N90 were therefore used to build a pathway map. The pathways that were affected include inflammation and reactive oxygen species (ROS) and the pentose phosphate pathway (PPP), which acts to balance oxidative stress ${ }^{27}$ (Figure $3 \mathrm{D}$ ). Together, these data indicate that nanovibrational stimulation triggers an energetic response in cells, as evidenced by increased glycolytic and tricarboxylic acid (TCA) cycle metabolite levels ${ }^{28}$ (Figure 3D). Metabolite pathways were also analyzed using ingenuity pathway analysis (IPA). The IPA analysis further supported these results. After 1 week of nanovibrational stimulation, metabolic pathways were upregulated in both N30 and N90 conditions, relative to control conditions, with greater upregulation seen in N90 conditions (supplementary Figure 3). By 2 weeks of nanostimulation, pathways were mostly downregulated in N30 conditions, but remained predominantly upregulated in N90 conditions (supplementary Figure 4). This, again, suggests that MSC osteogenesis stimulated by nanovibration is an energetic process and that the greater the stimulus, the greater the observed effects on the cell. We also note, from increases in ROS- and PPP-related metabolites, that redox balancing might also be potentially occurring to counter oxidative stress (Figure 3D).

To follow up the observation that redox balancing potentially occurs in response to nanostimulation, we again used IPA to analyze the metabolomic data. By looking at oxidative stress (Figure 4A), we observe pathways being increasingly activated in N90 relative to N30 conditions. Looking at the individual metabolites inferred as contributing toward oxidative stress, we observe the same metabolites forming the network, but being more highly activated in $\mathrm{N} 90$ conditions (Figure 4B,C). We next used 2'-7 dichlorodihydrofluorescein diacetate (DCF-DA) flow cytometry to measure ROS. While a small increase was noted in N30 conditions, relative to the control, this increase became statistically significant in N90 conditions (Figure 4D).

Previous studies have linked small increases in ROS to enhanced osteogenesis; however, large increases in ROS are also linked to the suppression of osteogenesis. ${ }^{29-31}$ To investigate this issue in our experimental system, we used $N$-acetyl cysteine to inhibit ROS (Figure 4E) and then assessed RUNX2 and OSX expression in MSCs after 9 days of culture by qPCR. Our results show that while RUNX2 and OSX were expressed at a low level in N30 conditions, both markers were significantly upregulated in N90 conditions, again showing that enhanced osteogenesis occurs with the larger amplitude. In addition, little change in marker expression was seen in response to ROS inhibition, indicating that ROS do not have a detrimental effect on osteogenesis and are a likely by-product, rather than a driver, of osteogenesis.

Using IPA activity predictor, where metabolite networks are linked to biochemical signaling hubs, some consistencies and some similarities and some differences in signaling could be observed between N30 and N90 stimulation (Figure 4F). ERK $1 / 2$ is predicted to be upregulated, as are the superoxide dismutase (SOD) pathways for both N30 and N90 stimulation. ERK $1 / 2$ stimulation is widely reported to be important for MSC osteogenesis, as it is implicated in the phosphorylation and activation of RUNX2, the osteogenic master transcription factor. ${ }^{32-34}$ SOD is used by cells to counter balance the effects of ROS, ${ }^{35}$ and so this finding fits with our observations of increased ROS production and PPP activation (Figure 4B,D). Interest- ingly, the results of stimulation at N90 also implicate the nuclear factor kappa-light-chain-enhancer of activated B cells $(\mathrm{NF} \kappa \mathrm{B})$ and protein kinase B (Akt) pathways, which are involved in cell survival and in preventing apoptosis and are both linked to antioxidant function. ${ }^{36,37}$

Inflammation and Nanovibrational Stimulation. Increased levels of ROS lead to inflammation, ${ }^{38}$ and a small degree of inflammation is implicated in the natural bone healing process, while high levels of inflammation prevent osteogenesis. $^{39}$ Given this, we hypothesized that inflammation might be observed in MSCs following their culture under N90 conditions. To explore this, we assayed the expression of the inflammatory markers, interleukin 6 (IL-6), NF $\kappa \mathrm{B}$, and tumor necrosis factor $(\mathrm{TNF} \alpha)$, by qPCR after 9 days of $\mathrm{N} 90$ culture, as well as that of two mitogen activated protein kinases (MAPKs), ERK (which is involved in cell proliferation and osteogenesis) ${ }^{32-34}$ and of c-jun n-terminal kinase (JNK, which is also implicated in osteogenesis ${ }^{32}$ but is better known for being activated by ROS or inflammation to mediate cytokines and apoptosis). ${ }^{40}$ We observed increased expression of TNF $\alpha$, ERK, and JNK after 9 days of culture and N90 stimulation compared to unstimulated control (Figure 5A).

We next inhibited these inflammatory pathways and p38 MAPK (which is activated by cell stress and is involved in apoptosis and differentiation control $)^{41}$ and assessed the expression of the osteogenic markers, RUNX2 and OSX, in MSCs cultured under N30 and N90 stimulation conditions. It was seen that compared to N30, N90 osteogenesis (RUNX2) was enhanced by $\mathrm{p} 38 \mathrm{MAPK}$ inhibition and reduced (OSX) by TNF $\alpha$ inhibition (Figure 5B).

Looking at the pro-inflammatory cytokine (IL-6) ${ }^{42}$ and the inflammation response factor $(\mathrm{NF} \kappa \mathrm{B})^{36}$ at days 7,14 , and 21 of MSC culture, we observed that the expression of these inflammatory mediators tracked each other; their expression increased to day 14 and then reduced (Figure 5C), most notably with N90. This suggests that nanostimulation induces an inflammatory response that is then countered by the cells and that the magnitude of this response scales with amplitude. We used ELISA to assess the levels of the major proinflammatory cytokine, IL- $1 \beta,{ }^{43}$ and found that it was undetectable at N30 or N90 conditions, with concentrations below the sensitivity of the standard curve (Figure 5D). This demonstrates that the inflammatory response we observed is small rather than constituting real inflammation, at a level more likely to be positive in terms of bone formation. ${ }^{39}$

Our unpublished data suggest that MSCs from $\sim 1$ in 20 donor samples do not respond to nanovibrational stimulation. We looked at $\mathrm{NF} \kappa \mathrm{B}$, which is linked to osteogenesis in MSCs, from one of these donors. In this donor, there was little evidence of osteogenesis having occurred after N30 or N90 stimulation, based on the expression of RUNX2, OSX, and OPN after 9 days of culture, (supplementary Figure $5 \mathrm{~A}$ ). However, when $\mathrm{NF} \kappa \mathrm{B}$ was inhibited with TPCA-1, the expected increase in these osteogenic markers was observed (supplementary Figure 5A). Furthermore, nanovibration resulted in the decreased expression of $\mathrm{NF} \kappa \mathrm{B}$ in direct proportion to vibration amplitude (supplementary Figure 5B). We thus speculate that the inflammatory background of the donor and/or dysregulation of $\mathrm{NF} \kappa \mathrm{B}$ might increase or decrease the osteogenic capacity of individual MSCs.

Tissue Engineering Using Nanovibration. These data demonstrate that increasing nanoscale amplitude can enhance osteogenesis through a low-level ROS/inflammatory axis in 3D 
A

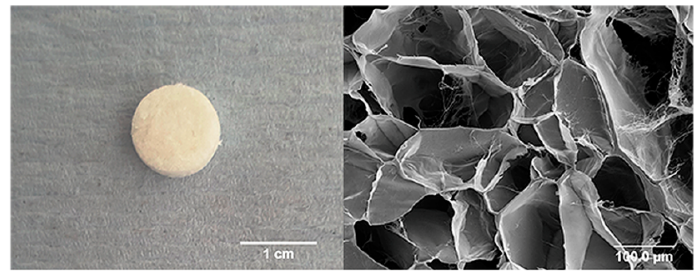

C

c

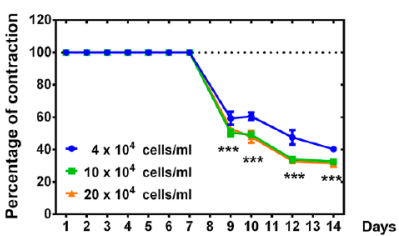

N30

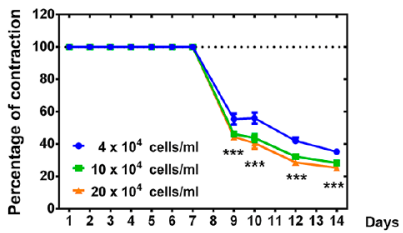

N90

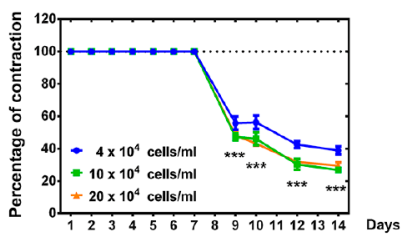

E

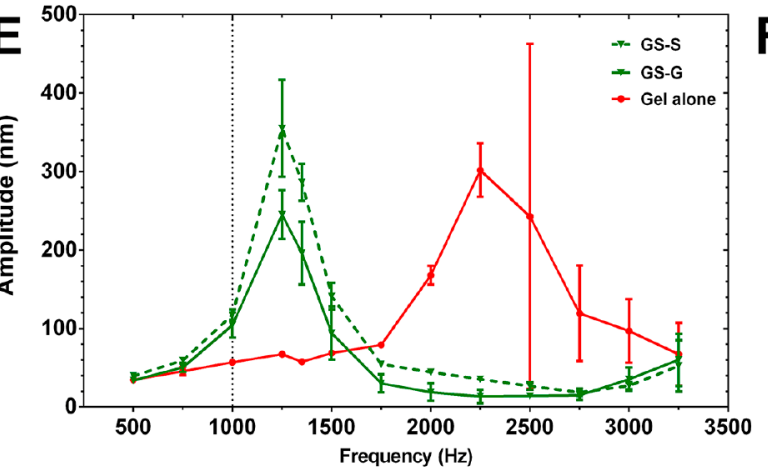

F

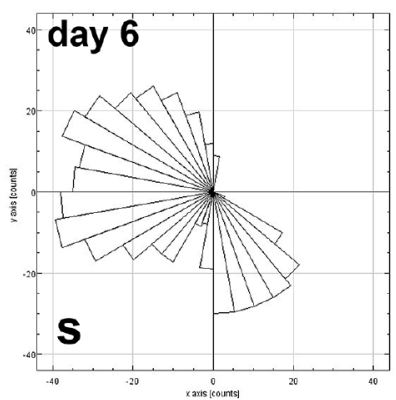

B

D
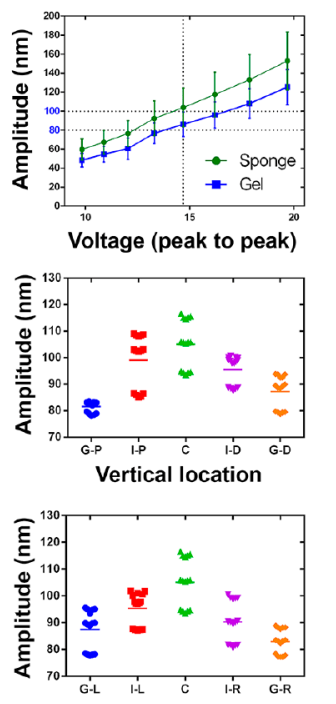

Horizontal location
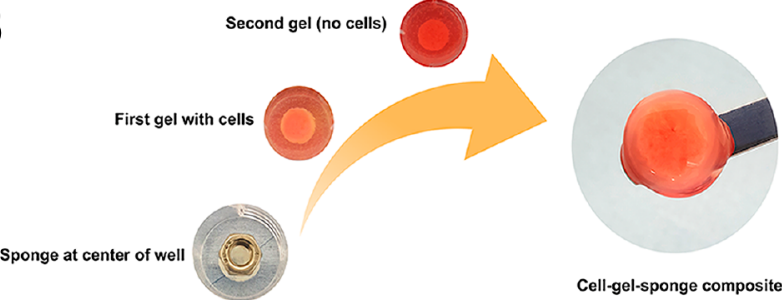

Cell-gel-sponge composite

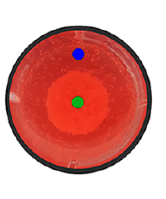

- Gel

- Sponge
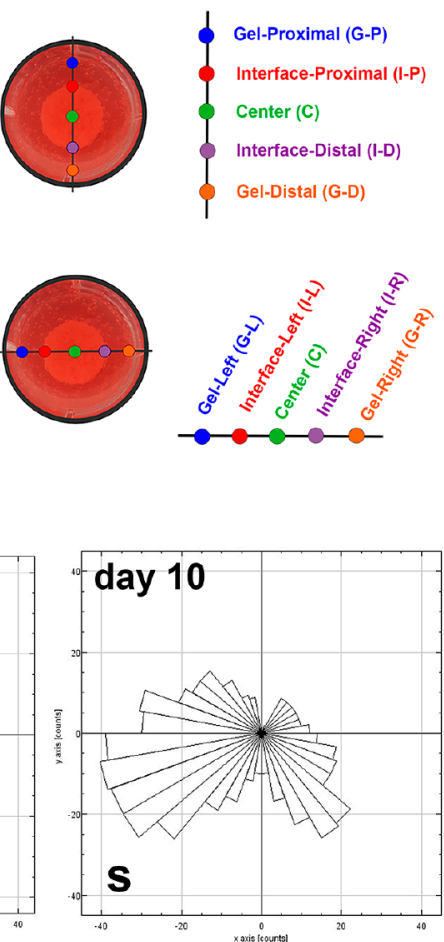

H
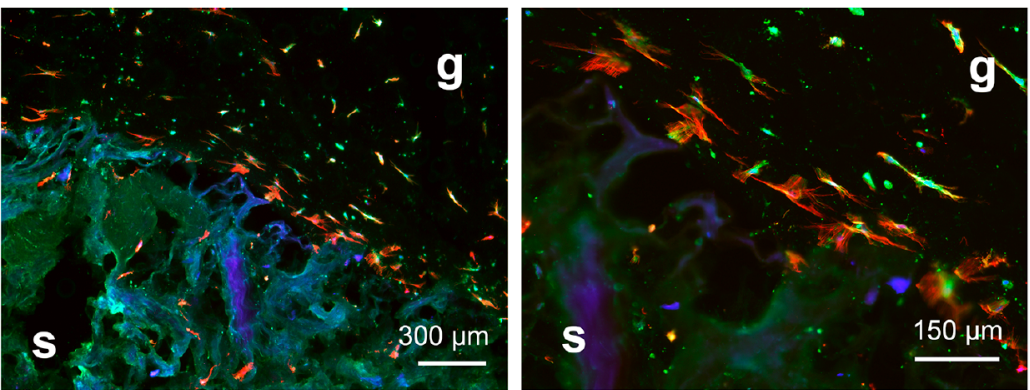

Figure 6. Design of a gel-sponge composite for nanovibrational stimulation. (A) A freeze-dried collagen sponge (left) showing its pore structure by SEM (right). (B) Schematic of the composite gel's fabrication. The sponge is held down with a weight, while the cell/gel mixture is poured over it and sets. After the weight is removed, more collagen is poured on. At a user-defined time, the cell-containing collagen gel can be released from the well edges and allowed to contract onto the sponge to form an easy to handle construct. (C) Gel constructs, containing 4, 10, and $20 \times$ $10^{4} \mathrm{MSCs} / \mathrm{mL}$, were stimulated for 7 days, and then the gels detached from the well. All gels contracted onto the sponge within 2 days, and the sponge prevented further contraction $(d=1, r=4, t=1)$. (D) Interferometry testing of vibrational transmission in the composite gel. A linear voltage amplitude relationship was seen both at the sponge and gel positions (top). Looking at points across the gel-sponge composites (middle and bottom), slightly higher amplitudes were observed at the sponge position compared to the gel at $\mathrm{N} 90$ stimulation, with up to $20 \mathrm{~nm}$ variance observed $(n=3-6)$. (E) Increasing vibration frequencies were assessed using interferometry. The sponge induced resonant frequencies at $\sim 1250 \mathrm{~Hz}$. At $1000 \mathrm{~Hz}$, however, little evidence of resonant effects was observed ( $n=3$, GS; gelsponge composite, G; gel area, $S$; sponge area). (F) MSC migration in the gel toward the sponge, shown at day 6 (precontraction) and at day 10 (post contraction). Movement toward the 
Figure 6. continued

sponge increased with contraction $(\mathrm{S}=$ sponge location). (G) Cell velocity, however, decreased postcontraction $(d=1, r=1, t=10)$. $(\mathrm{H})$ Histology of gel-sponge composite sections showing MSC migration into the gels $(\mathrm{red}=10$, green $=10$, blue $=10 ; \mathrm{g}=$ gel, $s=\mathrm{sponge})$. The data show that this fabricated gel-sponge composite can facilitate cell migration and the application of nanovibrational stimulation. Abbreviations: $d$ $=$ number of donors assessed; $r=$ number of wells tested; and $t=$ technical replicates.

A

RUNX2

osx

ALP

ON
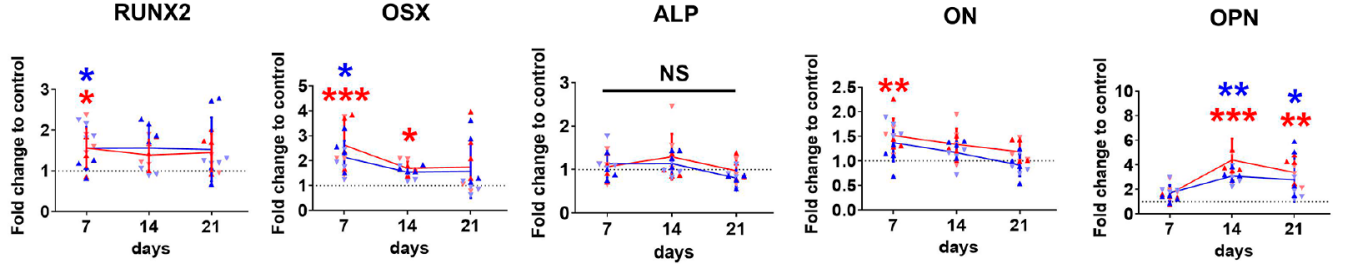

N30-Donor 1

N30-Donor 2

- N90-Donor 1

N90-Donor 2

B
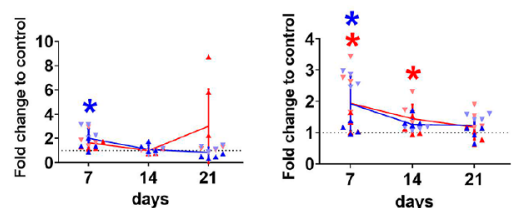

C

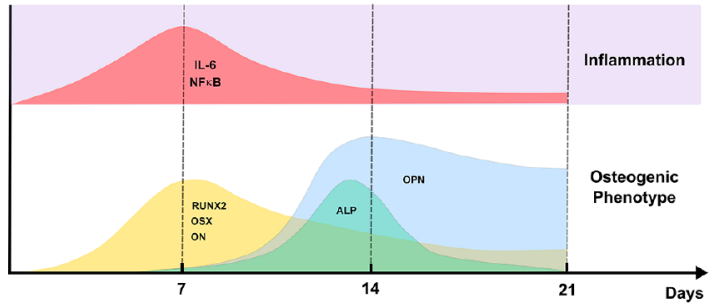

E

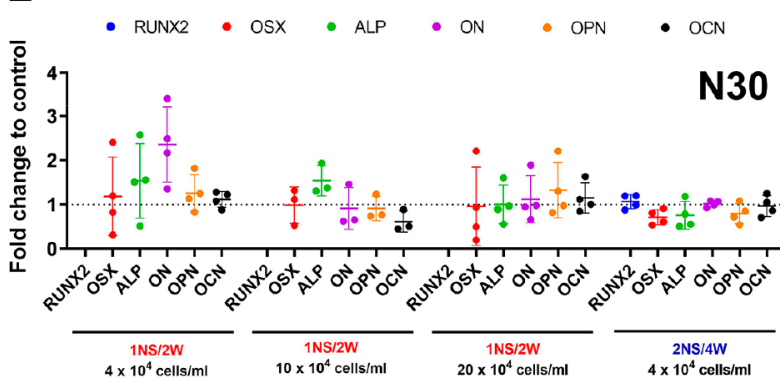

D

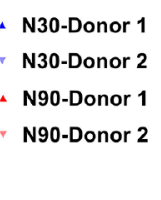

$\underset{4 \times 10^{4} \mathrm{cells} / \mathrm{ml}}{\mathbf{N} \mathrm{N} / 2 \mathrm{~W}}$

1NS/2W
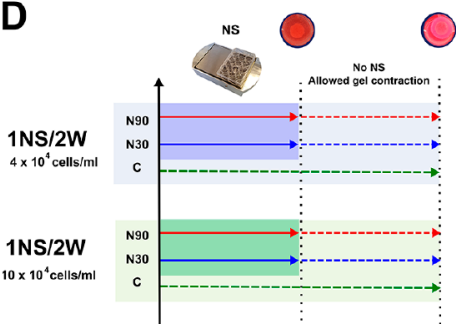

$\underset{20 \times 10^{4} \mathrm{cells} / \mathrm{ml}}{1 \mathrm{NS} / 2 \mathrm{~W}}$

$\bigcirc$
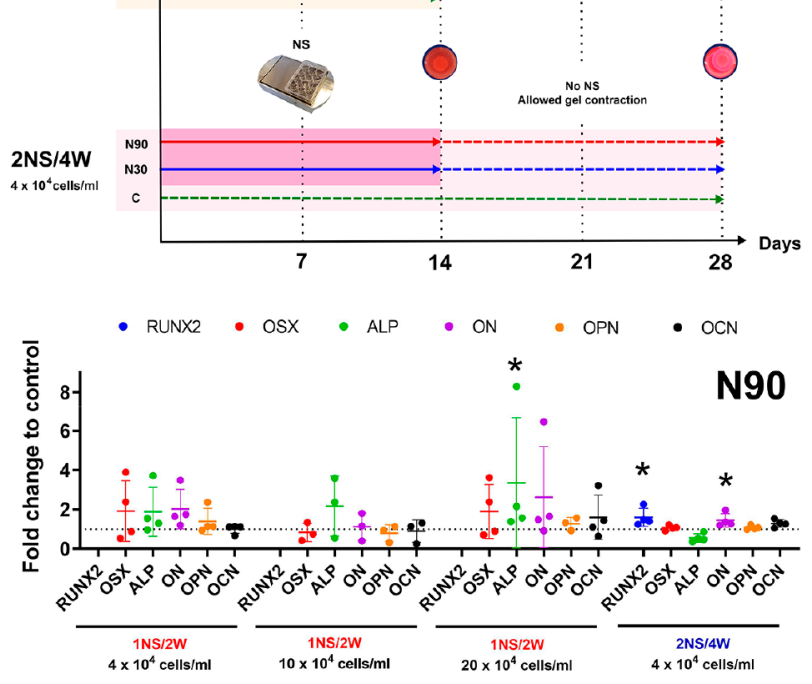

Figure 7. Osteogenesis and mechanical memory of gel-sponge constructs. (A) Osteogenic marker expression analysis by qPCR of MSCs cultured for 1, 2, and 3 weeks in the composite gel without gel detachment $(d=2, r=4, t=3)$. (B) IL-6 and NFKB inflammatory marker expression by $\mathrm{qPCR}$ of MSCs cultured for 1,2 , and 3 weeks in the composite gel without gel detachment. The markers are initially upregulated and then rapidly return to control levels $(d=2, r=4, t=3)$. (C) Schematic of the expression profiles of inflammatory and osteogenic markers in MSCs cultured under N90 stimulation. (D) Schematic of the stimulation, detachment, and experimental termination regimens for mechanical memory analysis. They were as follows: 1 week of nanostimulation followed by gel detachment from the sides of the well; 1 week of contraction $(1 \mathrm{NS} / 2 \mathrm{~W})$ for 4,10 , and $20 \times 10^{4}$ MSCs per $\mathrm{mL}$ of collagen, using N30 and N90 and 2 weeks of nanostimulation followed by gel detachment from the sides of the well; and 2 weeks of contraction (2NS/4W) for $4 \times 10^{4}$ MSCs per mL of collagen using N30 and N90. (E) Using N30, very little evidence of mechanical memory post-cessation of nanostimulation and subsequent contraction was observed. However, following N90 stimulation, evidence of mechanical memory was seen using $20 \times 10^{4} \mathrm{MSCs}$ per $\mathrm{mL}$ for the $1 \mathrm{NS} / 2 \mathrm{~W}$ regime with enhanced ALP expression. Mechanical memory was also observed using N90 for the 2NS/4W regime with $4 \times 10^{4}$ MSCs per mL with enhanced expression of RUNX2 and $O N(d=1, r=4, t=3)$. Error bars represent means $\pm S D$, significance calculated using ANOVA with Tukey multiple comparison where $*=p<$ $0.05, * *=p<0.01$, and $* * *=p<0.001$ (note that where applicable, blue and red asterisks show the significant difference of N30 and N90 to control, while black asterisks represents significant difference between N30 and N90). The data show that MSC osteo-differentiation occurs within the composite constructs and that, with N90, mechanical memory can be used to enable the production of a contracted, easy to handle, tissue-engineered product.

MSC cultures. Here, we look to see if these changes hold as we develop a composite more suitable to being used for tissue engineering.
The gels used in this report are very soft $(\sim 26-161 \mathrm{~Pa})$ and thus problematic to handle. Exploiting this osteogenic effect in a more structurally stable scaffold is of vital importance for the 
clinical translation of this technology. However, there are some constraints. The scaffold needs to be physically integrated with the well plate, and thus collagen gels are useful as they are biocompatible and attach to the sides and bottom of culture dishes (unless the cells detach the gel through contraction). Collagen gels are also highly hydrated. Water is incompressible, meaning that when constrained, such as in a culture plate well, it acts as a solid object. ${ }^{13}$ Hydrogels are mainly water, and this means that the cells experience vibration in all parts of the gel, as we demonstrate on top of the gels using interferometry (Figure 1D).

We thus decided to generate a composite gel using a collagen sponge to provide rigidity while maintaining biocompatibility. Insoluble collagen was freeze-dried to form a highly porous structure (pore size $227.74 \pm 72.93$, measured using Feret's diameter), ${ }^{44}$ which was $\sim 4 \mathrm{~mm}$ high and $\sim 11 \mathrm{~mm}$ in diameter (Figure 6A), and has an elastic modulus of $1.08 \pm 0.29 \mathrm{MPa}$ (Zwick-Roell compression testing). The acellular scaffolds were held in place with a small weight, while $2.5 \mathrm{~mL}$ of MSCcontaining neutralized collagen solution was poured over them. This sets the scaffolds within the wells of a 12 -well plate and provides a 3D MSC source (Figure 6B). Once the gel is set and the scaffold set in place, the weight is removed and a small amount of fresh collagen is added to complete the gel (Figure 6B).

In order to make scaffolds that are easier to handle for potential clinical applications, we utilized the adherent properties of collagen to allow for vibration fidelity. We did so by vibrating the gels for a period and then allowing the contractile properties of the cells in the collagen to pull the gels onto the sponge. To test this, gels were seeded with 4,10 , and $20 \times 10^{4}$ cells per mL of MSCs and cultured with/without N30 and N90 stimulation for 7 days. Gels were then released from the sides of the wells and contraction observed. All gels in all conditions contracted on to the sponge within 2 days, with $10 \times 10^{4}$ and 20 $\times 10^{4}$ MSCs contracting the gels more than $4 \times 10^{4}$ MSCs (Figure 6C). Next, interferometry was used to observe gel vibrational response to N90 input. We observed that vibration was higher in the well center (over the gel) at just over $100 \mathrm{~nm}$ and was lower at the well edges at $80-90 \mathrm{~nm}$ (Figure 6D); acceptable vibration fidelity was seen.

Using N90 conditions with $4 \times 10^{4} \mathrm{MSCs} / \mathrm{mL}$, we looked for resonant frequency at $1000 \mathrm{~Hz}$. While measuring at the center (sponge, S) and edge (gel, G) of the gel-sponge composite, no resonance effects were seen at increased amplitude of just over $100 \mathrm{~nm}$ (Figure 6E), enabling us to proceed with $1000 \mathrm{~Hz}$ stimulation.

We also looked at MSC migration into the gel composite at 4 $\times 10^{4} \mathrm{MSCs} / \mathrm{mL}$ without N90 stimulation. MSC migration, while always toward the sponge, became more targeted postcontraction (day 10) compared to pre-contraction (day 6) (Figure 6F). The velocity of MSC migration, however, decreased postcontraction (Figure $6 \mathrm{G}$ ). Histology at day 12 confirmed that cell migration into the gels had occurred (Figure $6 \mathrm{H})$.

We next moved to consider osteogenesis and also mechanical memory (or mechanical priming) of MSCs in the gel-sponge composites. This was in order to check first that the composites could be used with nanovibrational stimulation and then second to see if the composites could be vibrated for shorter-term cultures with prolonged osteogenesis (i.e., with memory of the initial vibration). Thus, in the gel-sponge composites, $4 \times 10^{4}$ MSCs/mL with/without $\mathrm{N} 30$ and N90 stimulation were assessed for osteogenic markers by qPCR after 1, 2, and 3 weeks of vibration without gel detachment. We observed the increased expression of pro-osteogenic markers at both N30 and N90 stimulation, and this increase was greater at N90 stimulation (Figure 7A). The expressions of IL- 6 and $\mathrm{NF} \kappa \mathrm{B}$ were also tested to assess the inflammatory response of MSCs during nanostimulation in the composite. Although IL-6 and $\mathrm{NF} \kappa \mathrm{B}$ were initially expressed, their expression quickly reduced to background levels (Figure 7B), concurring with our low-level inflammation-osteogenesis hypothesis (Figure 7C).

Finally, we assessed two different culture regimes for contracting the gel onto the sponge to enable the manufacture of free-floating scaffolds with good handling properties. The first used 1 week nanostimulation before gel detachment (osteogenic markers were upregulated in the MSCs in the composites) (Figure 7A) to produce free floating cell-composite scaffolds. Another week of culture was then allowed to promote full contraction onto the scaffold, giving a 2 week production timeline (1:1). This was performed with 4,10 , and $20 \times 10^{4}$ MSCs per $\mathrm{mL}$ of collagen. The second regime used 2 weeks of nanostimulation before gel detachment and a further 2 weeks of culture (2:2), giving a 4-week production timeline for the freefloating cell composites. For N30, very little osteogenesis was observed with either the $1: 1$ or $2: 2$ regimes (Figure $7 \mathrm{E}$ ). However, with N90, osteogenic transcriptional changes were observed for the 1:1 regime that used the highest level of cell loading $\left(20 \times 10^{4}\right)$ and with the longer 2:2 regime that used the lower cell loading level $\left(4 \times 10^{4}\right)$ (Figure $\left.7 \mathrm{E}\right)$.

\section{SUMMARY}

By increasing the amplitude of nanostimulation, we have been able to better dissect the cellular mechanisms of nanovibrational osteogenesis in MSCs. Many 2D osteogenesis studies have highlighted the central role of intracellular tension. In this 3D study, we can see clear changes in the regulation of integrin receptors and of ECM components, such as collagen (Figure 2A). We also observed the differential expression of known integrin-linked osteogenic pathways, such as ERK (Figure 2B). In all cases, stimulating MSCs with N90 produced a more marked transcriptional and protein-level response. However, reducing intracellular tension via ROCK inhibition did not significantly impact osteogenesis in our system (Figure 2D). Our results thus suggest that while adhesion is modulated and contributes to osteogenesis, it is not the central driver.

Several ion channels were also upregulated during nanovibrationally stimulated MSC osteogenesis. Ion channel expression and the number of channels showing enhanced expression were linked to increasing amplitude (Figure 2A,B). For example, at the protein level, the TRPV1 cation channel was upregulated in N30 conditions, while and TRPV1, TRPA1, Piezo 1 and 2 cation channels, L-type $\mathrm{Ca}^{2+}$ channel, and the KCNK2 potassium channel were upregulated in N90 conditions. The TRP cation channels are typically associated with temperature and pain sensing and can be activated via inflammatory mediators, such as IL-1, IL-6, and ROS, ${ }^{17,45,46}$ which we assess in this work. TRP and piezo channels have also been implicated in vibrational mechanotransduction, such as in sterocillia signaling in hearing. ${ }^{22}$ In fact, both TRP and piezo channels have also been linked to cytoskeletal organization, YES associated protein (YAP, a known osteogenic mechanoregulator), ${ }^{47,48}$ and BMP-2 regulated osteogenesis. ${ }^{24,49,50}$ This ties in well with previous findings that $3 \mathrm{D}$ osteogenesis can be disrupted with TRPV1 inhibitors ${ }^{9}$ and adds to the body of evidence suggesting that 


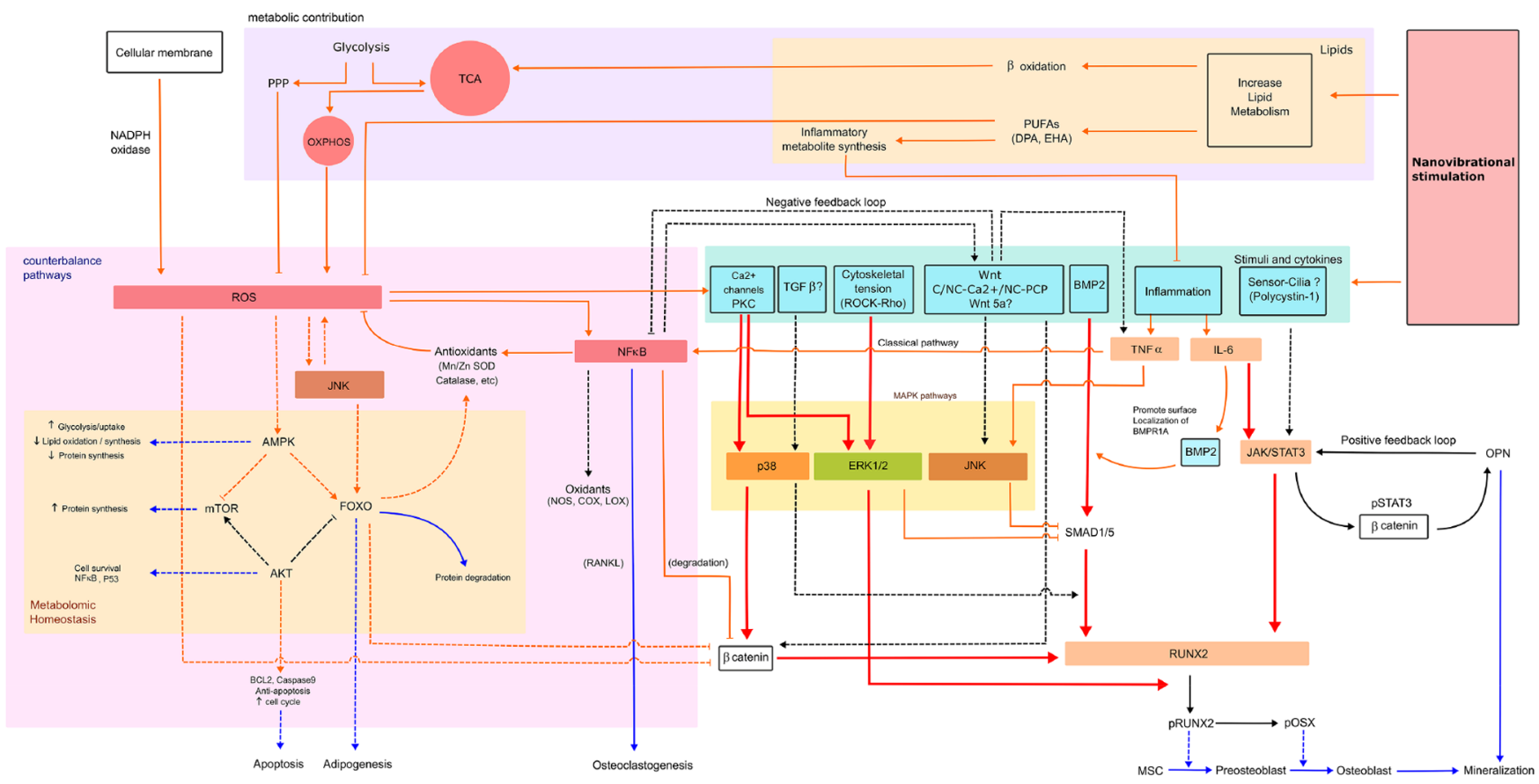

Figure 8. A model of the mechanisms that contributes to nonvibrational MSC stimulation illustrating that respiration is linked to ROS and inflammation balancing responses and MAPK signaling to drive osteogenesis. Abbreviations: AMPK $=\mathrm{AMP}$-activated protein kinase, $\mathrm{AKT}=$ protein kinase B (PKB), BCL2 = B-cell lymphoma 2, C-Wnt = canonical Wnt, COX = cyclooxygenase, FOXO = forkhead box class O, JAK = Janus kinases, $\mathrm{LOX}=$ lipoxygenase, $\mathrm{mTOR}=$ mammalian target of rapamycin, $\mathrm{NADPH}$ oxidase = nicotinamide adenine dinucleotide phosphate oxidase, $\mathrm{NC}-\mathrm{Ca}^{2+}=$ noncanonical Wnt/calcium pathway, NC-PCP = noncanonical planar cell polarity pathway, NOS = nitric oxide synthase, OXPHOS = oxidative phosphorylation, $\mathrm{PKC}=$ protein kinase $\mathrm{C}, \mathrm{pOSX}=$ phosphorylated osterix, $\mathrm{pSTAT3}=$ signal transducer and activator of transcription protein $3, \mathrm{RANKL}=$ receptor activator of nuclear factor kappa-B ligand, SMAD1/5 = small mothers against decapentaplegic homologue 1/5, and Wnt5a = wingless-related integration site. Line description, red line; expected high-level expression, orange line; expected low-level expression, blue line; phenotype expression, solid line; predicted pathways, dotted line; theoretical pathways.

these ion channels have wide ranging effects in cellular mechanotransduction. Again, subtle results observed at N30 were more evident at $\mathrm{N} 90$.

The transduction of higher frequency sound waves into biologically relevant signals is a poorly understood mechanism. However, we postulate that cells might convert higher frequency stimuli in constant excitatory responses. For example, at low frequency, Piezol behaves like a bandpass filter with a center frequency at around $10 \mathrm{~Hz} .{ }^{51}$ At higher frequencies, the in-phase peak response disappears, and a "tonic" current remains, which in turn increases with frequency. This model predicts that for a $1000 \mathrm{~Hz}$ stimulus, a sustained tonic current is expected, which might be comparable with a single stimulus peak response when the number of stimulated channels exceeds several hundred. ${ }^{51}$ This behavior suggests the presence of an underlying molecularlever mechanism that is able to transduce the mechanical stimulus with a frequency-dependent efficiency. While the specific origin of this mechanism is not yet clear, it is noteworthy that many mechanosensitive ion channels have been found to have common structural features, and it is likely that this is the root for a broad and concerted cell mechanosensitivity. ${ }^{52}$

Untargeted metabolomics analysis led us to look at ROS and inflammation. Subtle increases in ROS and redox-balancing pathways, such as PPP and SOD, were activated in response to nanostimulation at N90 (Figures 3C and 4). Similarly, proinflammatory and inflammation-mediating pathways were transcriptionally activated (Figure 5). However, pro-inflammatory cytokines were not detected at the protein level following MSC stimulation at N90 (Figure 5D). Our metabolomic data also reveal hallmarks of increased energy demand during MSC differentiation, which concurs with the literature showing that increased oxidative phosphorylation occurs in MSCs undergoing differentiation to produce more ATP. ${ }^{36,53,54}$ From these findings, we propose that this energy demand drives increased levels of ROS and thus some of the markers of inflammation. It is well-known that ROS is generated via increased electron transport chain activity. ${ }^{55}$ However, MSCs counter this increase in potentially damaging pathways. ${ }^{31}$ Inhibiting ROS had only a small effect on osteogenesis (Figure 4E) and likely illustrates that ROS production and inflammation are byproducts of osteogenesis rather than drivers of it. Early stage, low level increases in ROS and inflammation are seen in the earliest stages of bone fracture repair-in the inflammatory phase, also known as fracture hematoma formation. ${ }^{39}$ From our data, we speculate that this inflammation is a byproduct of bone cell stimulation. Together, our metabolomic and biochemical data, and those already published, lead us to propose a model for nanovibrational stimulation pathways, in which respiration/energy are linked to ROS and inflammation balancing responses and MAPK signaling to drive osteogenesis (Figure 8). However, more work is required to fully elucidate these proposed pathways.

Our data also allow us to speculate that over a threshold amplitude, nanovibrational stimulation could become detrimental to cells as they struggle to balance the increasing levels of ROS and inflammation. Furthermore, our analysis of a nonresponding donor MSC line indicates that inflammatory background might be important in the selection of donor material for orthopedic cell manufacture (supplementary Figure 5). 
Finally, our study explored the effects of both an increased N90 amplitude on MSC osteogenesis and also the generation of a sponge-gel composite that is easier to handle. We assessed MSCs cultured in this gel composite for markers of osteogenesis and inflammation and observed enhanced osteogenesis in MSCs cultured under N90, relative to N30 conditions, and also some initial expression of IL- 6 and $\mathrm{NF} \kappa \mathrm{B}$ that quickly reduced. This lends further support to the hypothesis that low-level inflammation occurs in nanovibration-enhanced osteogenesis. We also investigated when we could allow the gels to contract onto the sponge to minimize manufacturing time for use in the bioreactor and found that 2 weeks of $\mathrm{N} 90$ treatment provide the most optimal time.

\section{CONCLUSIONS}

In this study, we demonstrate the important contribution of amplitude to the nanovibrational stimulation of osteogenesis in MSCs. We find that an amplitude of N90 produces increased osteogenesis, relative to an amplitude of N30, and use these different responses to investigate subtle changes in adhesion, tension, ion channel regulation, ROS, and inflammation in the osteogenic MSC response. Using our bioreactor, we provide insights into the low-level ROS and inflammatory responses that are typically seen with osteogenesis both in culture, and in the clinic, resulting from the energetic demands of differentiation. We confirm these findings in a bioengineered 3D osteogenic cellular composite, which works with our bioreactor and provides both an enhanced osteogenic environment and handlability.

\section{METHODS}

Cell Culture. Stro- 1 selected MSCs from adult human bone marrow (BM) with informed consent from Southampton General Hospital. MSCs were cultured in Dulbecco's modified essential medium (DMEM; Sigma) supplemented with 10\% fetal bovine serum (FBS; Sigma), 1\% (v/v) L-glutamine (200 mM, Gibco), 1\% sodium pyruvate (11 mg/mL, Sigma), 1\% MEM NEAA (amino acids, Gibco), and 2\% antibiotics $(6.74 \mathrm{U} / \mathrm{mL}$ penicillin-streptomycin, $0.2 \mu \mathrm{g} / \mathrm{mL}$ fungizone; Sigma). MSCs were cultured in an incubator set at $37^{\circ} \mathrm{C}$ with $5 \% \mathrm{CO}_{2}$ environment and subcultured to passage $2-3$ before use. Culture media was changed every 3 days.

Hydrogel Preparation. 0.8 and $1.8 \mathrm{mg} / \mathrm{mL}$ collagen hydrogels were prepared using rat tail collagen type I ( 2 and $5 \mathrm{mg} / \mathrm{mL}$, First link, UK). $10 \times$ DMEM (First link, UK) and FBS (Sigma) were added as a cell supplement. $0.1 \mathrm{M}$ sodium hydroxide (Fluka, UK) was used for $\mathrm{pH}$ titration to achieve a $\mathrm{pH}$ of 7.7-8.0 judged by universal litmus paper and phenol red indicator. After $\mathrm{pH}$ titration, Stro- 1 selected MSCs were added in to the hydrogel mixture (final cell concentration was $4 \times 10^{4}$ cells per $\mathrm{mL}$ of hydrogel) and decanted as $2.5 \mathrm{~mL}$ pregels into 6- or 12well plates. Gelation followed in a $37^{\circ} \mathrm{C}$ incubator for $30 \mathrm{~min}$.

Bioreactor Set up. The nanovibrational bioreactor incorporating piezo actuators (Thru-ring actuators; P-010.00H, Physik Instrumente, Germany) was used to stimulate cell cultures. A laptop, as signal generator, was connected to the amplifier (Linn amplifier, Sneaky DS, UK) transferring an electrical signal to the nanovibrational bioreactor causing piezo expansion. Selected nanovibrational stimulation (NS) frequencies in a.flac file type were operated on Kinsky software (Version 4.3.14), using as a file control panel. To construct the culture platebioreactor apparatus, adhesive magnetic sheets $(3 \mathrm{M}, \mathrm{UK})$ were adhered to the bottom of 6- or 12-well plates (Corning, USA) and attached on the platform of the bioreactor.

Freeze-Dried Collagen Sponge Preparation. To prepare the collagen sponges, a $5 \%$ weight of bovine tendon powder (Collagen Solutions, UK) was used. $0.001 \mathrm{M}$ of $\mathrm{HCl}$ at $\mathrm{pH} 3$ was added and then homogenized (TissueRuptor, Qiagen) on ice. The mixed composites were then molded in polystyrene cell culture inserts $(0.4 \mu \mathrm{m}$ polyethylene terephthalate (PET) membrane, 12-well plate diameter, Greiner bio-one, Austria) and in turn frozen at $-80^{\circ} \mathrm{C}$ for $10 \mathrm{~h}$. Freezedrying was performed at $-110^{\circ} \mathrm{C}$ with a vapor pressure at $0 \mathrm{mbar}$, for $20 \mathrm{~h}$ (VirTis, SP industries, USA). Freeze-dried collagen sponges were sterilized by UV light exposure for $1 \mathrm{~h}$.

Collagen-Hydrogel Sponge Composite Preparation. To prepare the composites, sponges were placed at the center of 12-well plates and were weighed down by placing a sterile screw nut on top of the sponges. $1.8 \mathrm{mg} / \mathrm{mL}$ collagen hydrogels were prepared, and $2.5 \mathrm{~mL}$ of hydrogel with MSCs was poured around the sponges. Gelation was allowed at $\mathrm{pH} 7.7-8.0$ in a $37^{\circ} \mathrm{C}$ incubator. Following the cell-hydrogel gelation, $0.5 \mathrm{~mL}$ of secondary hydrogels $(1.8 \mathrm{mg} / \mathrm{mL})$ without cells were aliquoted on top of the composite to fill any gaps. The composites were then transferred for stimulation on the NS bioreactor. When the stimulation time ended, the composites were detached from the culture wells by spatula, allowing contraction before removal for further analysis.

Scanning Electron Microscopy. The samples were prepared for SEM using critical point drying and sputtering. The samples were mounted onto SEM stubs using double-sided conductive tape and silver paint. They were then coated with gold/palladium (approximately 10$20 \mathrm{~nm}$ ) using a SEM coating system (Q150T ES, Quorum, UK). The samples were viewed on a JEOL6400 SEM running at $10 \mathrm{kV}$. Porosity diameter was analyzed by ImageJ (free download from $\mathrm{NIH}$ ).

Composite Contraction Measurement and Time Lapse Microscopy. In order to monitor the hydrogel contraction for the composites without NS, the percentage of the hydrogel contraction compared to the initial surface area was measured from a top view. The surface areas were measured and analyzed by ImageJ software. To evaluate cell migration, composites were cultured for 6 days precontraction and 10 days postcontraction. The composites were then imaged using a $10 \times$ objective lens at $120 \mathrm{~s}$ intervals for $24 \mathrm{~h}$ at 37 ${ }^{\circ} \mathrm{C}$. Cell velocity and migration directions were analyzed using image plugin (manual tracking) and chemotaxis and migration tool (Ibidi).

Cryosection and Immunostaining. After composites were stimulated with NS, the samples were fixed in $4 \%$ formadehyde and infused in $30 \%$ sucrose in PBS for cryoprotection overnight at $4{ }^{\circ} \mathrm{C}$. The samples were then transferred to embedding molds, and optimal cutting temperature (O.C.T.) embedding compound (Tissue-Tek, Sakura) was aliquoted to cover the samples. The samples were then frozen in liquid nitrogen and kept at $-80^{\circ} \mathrm{C}$. The frozen samples were sliced into $60 \mathrm{~mm}$-thickness sections and attached onto adhesive slides (9597, Tissue-Tek, Sakura, Netherlands). Immunofluorescent staining was then carried out. Samples were rinsed with $1 \times$ PBS and fixed with fixative at $37{ }^{\circ} \mathrm{C}$ for $15 \mathrm{~min}$. After that, permeabilization buffer was added and incubated at $4{ }^{\circ} \mathrm{C}$ for $5 \mathrm{~min}$. Samples were then blocked with $1 \%$ BSA in $1 \times$ PBS at $37^{\circ} \mathrm{C}$ for $5 \mathrm{~min}$. Primary antibody (P-myosin light chain 2, cell signaling, 3671S, rabbit, 1:50) and rhodaminephalloidin (Invitrogen, Thermo Fisher, 1:500) diluted in 1\% BSA in $1 \times$ PBS were added and incubated at $37{ }^{\circ} \mathrm{C}$ for $1 \mathrm{~h}$. Samples were then washed with $1 \times \mathrm{PBS} / 0.5 \%$ Tween-20 for 3 times ( 5 min each). Biotinylated secondary antibody (antirabbit; Vector Laboratories, USA, 1:50) diluted in $1 \%$ BSA in $1 \times$ PBS was added and incubated at $37^{\circ} \mathrm{C}$ for $1 \mathrm{~h}$. Samples were washed again with $1 \times \mathrm{PBS} / 0.5 \%$ Tween for 3 times. Streptavidin-FITC (Vector Laboratories, USA, 1:50) diluted in $1 \% \mathrm{BSA}$ in $1 \times \mathrm{PBS}$ was incubated at $4{ }^{\circ} \mathrm{C}$ for $30 \mathrm{~min}$. Samples were washed for 3 times. A small drop of 4',6-diamidino-2 phenylindole (DAPI, Vectashield) was placed onto the samples and covered with coverslips. FITC/TRITC channel images were taken (Olympus, US) operated on Surveyor software version 9.0.1.4 (Objective Imaging, UK). Images were processed using Image (Version $1.50 \mathrm{~g}$, USA) and Photoshop CS4 (Adobe, version11 extended, Ireland).

Interferometric Measurement. 0.8 or $1.8 \mathrm{mg} / \mathrm{mL}$ collagen hydrogels were prepared. The hydrogel nanovibration was measured by laser interferometric vibrometer (wavelength $=632.8 \mathrm{~nm}, \mathrm{CW}$ power; $5 \mathrm{~mW}$; SIOS, Me $\beta$ technik $\mathrm{GmbH}$, Germany). To reflect the laser beam, $3 \times 3 \mathrm{~mm}$ of reflective tape was placed on the hydrogels surface underneath $1.5 \mathrm{~mL}$ of media. The vibration distance was analyzed using INFAS Vibro 1.8.4 software (SIOS, Me $\beta$ technik GmbH, Germany). 
Rheology. To investigate the viscoelastic properties of hydrogels, a modular compact rheometer (MCR 302, Anton Paar, Austria) equipped with parallel plates of $25 \mathrm{~mm}$ diameter was used. Measurements were performed at a temperature of $23{ }^{\circ} \mathrm{C}$, under a constant normal force and gap size both ranging from 0.1 to $0.3 \mathrm{~N}$ and from 1.4 to $2 \mathrm{~mm}$, depending on the samples, respectively. Initial strain sweep tests at a constant frequency of $10 \mathrm{rad} / \mathrm{s}$ were performed to determine the range of linear response of the hydrogels. Then, the hydrogels' linear viscoelastic properties were measured by means of frequency sweep tests performed with a constant strain amplitude (of ca. $1 \%$ for most of the samples) and frequencies ranging from 100 to 0.1 $\mathrm{rad} / \mathrm{s}$.

AlamarBlue Assay. Four $\times 10^{4}$ cells $/ \mathrm{mL}$ of Stro- 1 selected MSCs were prepared in hydrogels or composites and stimulated for 1 and 2 weeks. Samples were washed with warm $1 \times$ PBS. $10 \%(\mathrm{v} / \mathrm{v})$ of AlamarBlue resazurin (Bio-Rad, Watford, UK) was diluted in phenolred free media (D5030, Sigma). After incubation at $37^{\circ} \mathrm{C}$ and $5 \% \mathrm{CO}_{2}$ for $5 \mathrm{~h}$, the supernatant containing the taken up AlamarBlue was pipetted and transferred into 96-well plates. A microplate reader (Clariostar, BMG Labtech, Germany) was used to detect light absorbance at wavelengths of 570 and $600 \mathrm{~nm}$. The percentage of AlamarBlue reduction was calculated as per ref 56 .

Quantitative Polymerase Chain Reaction with Reverse Transcription. To assess gene expression changes, $4 \times 10^{4}$ cells $/ \mathrm{mL}$ of hydrogel were prepared for qRT-PCR. Samples were removed from the bioreactor and transferred to falcon tubes. $2.5 \mathrm{mg} / \mathrm{mL}$ of collagenase (Sigma-Aldrich, UK) was added and incubated for $1.5 \mathrm{~h}$

Table 1. Thermal Cycler Protocol for cDNA Synthesis

\begin{tabular}{|lcc|}
\hline \multicolumn{1}{c}{ reaction } & temperature $\left({ }^{\circ} \mathrm{C}\right)$ & times $(\mathrm{min})$ \\
\hline genomic DNA elimination & 42 & 2 \\
reverse transcription & 42 & 30 \\
inactivation & 95 & 3
\end{tabular}

to digest the collagen hydrogels. Trizol (Life Technologies) and chloroform (Sigma-Aldrich, UK) were then added with ratio 5:1 in order to purify nucleic acids. An RNA extraction kit (RNeasy extraction Kit, Qiagen) was used to purify RNA. The concentration of purified RNA was measured by spectrophotometer (Nanodrop 2000c, Thermo scientific, USA). cDNA was then synthesized using the QuantiTect Reverse Transcription Kit (Qiagen). The cycling temperature in each process is shown in Table 1. Forward and reverse primers for qRT-PCR are shown in Table 2. GAPDH, a house-keeping gene, was used as internal control of the analysis. SYBR Green dye was used to target synthesized cDNA (Quantifast SYBR Green I, Qiagen). Real-time PCR was then performed (7500 Real Time PCR system, Applied Biosystem, USA). $2^{-\Delta \Delta C T}$ method was used for interpretation.

Inhibitor Studies. Four $\times 10^{4}$ cells $/ \mathrm{mL}$ of Stro 1 selected MSCs in $1.8 \mathrm{mg} / \mathrm{mL}$ collagen hydrogels were stimulated with N30 and N90 for 9 days. Inhibitors, which were diluted in basal media to working concentration, were added at day 2 (list of inhibitors and used concentration are shown in Table 3). Culture media with diluted inhibitor was changed every 2 days.

Protein Antibody Microarrays. To manufacture the arrays, commercial antibodies (Table 4) were buffer exchanged into PBS and quantified by bicinchoninic acid (BCA) assay. Antibodies were diluted to print concentration in PBS and printed in six replicates on Nexterion $\mathrm{H}$ amine reactive hydrogel-coated glass slides (Schott AG, Mainz, Germany) using a SciFLEXARRAYER S3 piezoelectric printer (Scienion, Berlin, Germany) under constant humidity $(62 \% \pm 2 \%)$ at $20^{\circ} \mathrm{C}$. Each feature was printed using $\approx 1 \mathrm{~nL}$ of diluted antibody via an uncoated $90 \mu \mathrm{m}$ glass nozzle with eight replicated subarrays per microarray slide. After printing, slides were incubated in a humidity chamber overnight at room temperature to facilitate complete conjugation. The slides were then blocked in $100 \times 10^{-3} \mathrm{M}$

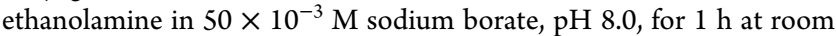
temperature. Slides were washed in PBS with $0.05 \%$ Tween 20 (PBS-T) three times for $2 \mathrm{~min}$, each wash followed by one wash in PBS, dried by centrifugation $(470 \times g, 5 \mathrm{~min})$, and then stored with desiccant at $4{ }^{\circ} \mathrm{C}$

Table 2. Primer Sequences Used in qRT-PCR

\begin{tabular}{|c|c|c|}
\hline & forward primer & reverse primer \\
\hline ALP & ATGAAGGAAAAGCCAAGCAG & CCACCAAATGTGAAGACGTG \\
\hline BMP2 & CССACTTGGAGGAGAAACAA & AGCCACAATCCAGTCATTCC \\
\hline CaSR & CTACGCACCAGAACTCCCTG & CTGTAACAGTGCTGCCTCCA \\
\hline COL1A1 & CCATGTGAAATTGTCTCCCA & GGGGCAAGACAGTGATTGAA \\
\hline ERK1 & CССTAGCCCAGACAGACATC & GCACAGTGTCCATTTTCTAACAGT \\
\hline ERK2 & TCTGCACCGTGACCTCAA & GCCAGGCCAAAGTCACAG \\
\hline GAPDH & TCAAGGCTGAGAACGGGAA & TGGGTGGCAGTGATGGCA \\
\hline IL-6 & GATGAGTACAAAAGTCCTGATCCA & CTGCAGCCACTGGTTCTGT \\
\hline Integrin $\beta 5$ & CTGTGGTCGGTAGCATCCTC & GGATCGCTCGCTCTGAAACT \\
\hline Integrin $\beta 1$ & GTGCAATGAAGGGCGTGTT & GTTGCACTCACACACACGACA \\
\hline JNK1 & GGGCAGCCСTCTCCTTTA & CATTGACAGACGACGATGATG \\
\hline JNK2 & GAAAGAAGCAAGAATGGTGTTGT & GAGAAGGAGTGGCGTTGCTA \\
\hline $\mathrm{NF} \kappa \mathrm{B}$ & CAGCTGGCTGAAGATGTGAA & GTGTTTTGGAAGGAGCAGGA \\
\hline $\mathrm{NF} \kappa \mathrm{B}$ & CAGCTGGCTGAAGATGTGAA & GTGTTTTGGAAGGAGCAGGA \\
\hline OCN & CAGCGAGGTAGTGAAGAGACC & TCTGGAGTTTATTTGGGAGCAG \\
\hline OSN & AGAATGAGAAGCGCCTGGAG & CTGCCAGTGTACAGGGAAGA \\
\hline OPN & AGCTGGATGACCAGAGTGCT & TGAAATTCATGGCTGTGGAA \\
\hline osterix & GGCAAAGCAGGCACAAAGAAG & AATGAGTGGGAAAAGGGAGGG \\
\hline $\operatorname{PPAR} \gamma$ & TGTGAAGCCCATTGAAGACA & CTGCAGTAGCTGCACGTGTT \\
\hline Piezo1 & TCGCTGGTCTACCTGCTCTT & GGCCTGTGTGACCTTGGA \\
\hline Piezo2 & CCCGGAGTTTGAAAATGAAG & CAGTGCCTCTTCTGAATCAATTT \\
\hline RUNX2 & GGTCAGATGCAGGCGGCCC & TACGTGTGGTAGCGCGTGGC \\
\hline SMAD1 & CCACTATAAGAGAGTAGAAAGCCCTGT & AAGTTACGGAACTGAGCTAAGAGG \\
\hline SMAD5 & GGGTGCCATGGAGGAACTGGA & AATCCGGCCAGCGCCAAACA \\
\hline TNF $\alpha$ & CAGCСТСТTСТССТTССТGAT & GCCAGAGGGCTGATTAGAGA \\
\hline TRPA & TGGACACCTTCTTCTTGCATT & TCTTCTCCATTAGCTCAATTTGG \\
\hline TRPV1 & AGAGTCACGCTGGCAACC & GGCAGAGACTCTCCATCACAC \\
\hline
\end{tabular}


Table 3. List of Inhibitors and Working Concentration

\begin{tabular}{clcc} 
function & \multicolumn{1}{c}{ inhibitors } & cat no./batch & $\begin{array}{c}\text { working } \\
\text { concentration }\end{array}$ \\
$\begin{array}{c}\text { ERK } \\
\text { inhibitor }\end{array}$ & U126, Tocris & $1144 / 5$ & $10 \mu \mathrm{M}$ \\
$\begin{array}{c}\text { P38 } \\
\text { inhibitor }\end{array}$ & SB 202190, Tocris & $1264 / 5$ & $5 \mu \mathrm{M}$ \\
$\begin{array}{c}\text { JNK } \\
\text { inhibitor }\end{array}$ & SP600125, Tocris & $1496 / 10$ & $25 \mu \mathrm{M}$ \\
$\begin{array}{c}\text { NFkB } \\
\text { inhibitor }\end{array}$ & TPCA-1, Tocris & $2559 / 5$ & $5 \mu \mathrm{M}$ \\
$\begin{array}{c}\text { TNF alpha } \\
\text { ROCK } \\
\text { inhibitor }\end{array}$ & $\begin{array}{c}\mathrm{R} \text { 7050, Tocris } \\
\text { Y-27632, Tocris }\end{array}$ & $5432 / 1$ & $2 \mu \mathrm{M}$ \\
$\begin{array}{c}\text { ROS } \\
\text { inhibitor }\end{array}$ & $\begin{array}{c}\text { N-acetyl cysteine, } \\
\text { Sigma-Aldrich }\end{array}$ & WXBC4028V & $10 \mu \mathrm{M}$ \\
& & &
\end{tabular}

Hydrophilic interaction liquid chromatography-mass spectrometry was performed (Dionex, UltiMate 3000 RSLC system, Thermo Fisher Scientific, Hemel Hempstead, UK) using a ZIC-pHILIC column (150 $\mathrm{mm} \times 4.6 \mathrm{~mm}, 5 \mu \mathrm{m}$ particle size, Merck Sequant). The data sets were processed using XCMS (peak picking), MzMatch (filter and grouping), and IDEOM (post processing filtering and identification). Metaboanalyst was used to generate heatmaps and PCA analysis. KEGG database and Ingenuity Pathway Analysis (IPA) software were used for metabolomic pathway analysis.

Reactive Oxygen Species Measurement. Stro-1-selected MSCs seeded with $4 \times 10^{4}$ cells $/ \mathrm{mL}$ density in $1.8 \mathrm{mg} / \mathrm{mL}$ collagen hydrogels were stimulated with N30 and N90 for 7 days. The samples were incubated in $2.5 \mathrm{mg} / \mathrm{mL}$ collagenase for $1 \mathrm{~h}$ and were then centrifuged at $200 \times g$ for $4 \mathrm{~min}$. Following that, the cell pellets were incubated for an hour in $2 \mu \mathrm{M} \mathrm{2} 2^{\prime}, 7^{\prime}$ - dichlorodihydrogen-fluorescein diacetate $\left(\mathrm{H}_{2} \mathrm{DCF}-\mathrm{DA}\right.$, Invitrogen) in phenol red free media (Sigma, D5030). In the positive control group, $500 \mu \mathrm{M}$ hydrogen peroxide was added. After

Table 4. List of Commercial Antibodies Used in the Protein Antibody Microarray

\begin{tabular}{|c|c|c|c|c|}
\hline probe & concentration $(\mathrm{mg} / \mathrm{mL})$ & company & stock conc $(\mathrm{mg} / \mathrm{mL})$ & cat. no. \\
\hline Integrin $\beta 1$ & 1 & Abcam & 3.54 & ab134179 \\
\hline Integrin $\beta 3$ & 0.1 & Abcam & 0.17 & ab34409 \\
\hline Integrin $\beta 5$ & $<0.002$ & Cell signaling & $<0.002$ & D24A5 \\
\hline BMPR1A & 1 & ThermoFisher & 12.71 & PA5-11856 \\
\hline Collagen I & 1 & Abcam & 1.72 & ab138492 \\
\hline Collagen II & 0.25 & Abcam & 2.29 & ab185430 \\
\hline Collagen III & 0.5 & Abcam & 1.03 & $\mathrm{ab} 7778$ \\
\hline Collagen V & 1 & Abcam & 1.86 & $a b 7046$ \\
\hline KCNK2 & 1 & SantaCruz & 4.84 & sc- 11557 \\
\hline KCNK4 & 0.02 & Abcam & 0.02 & ab81367 \\
\hline TRPA1 & 0.5 & SantaCruz & 5.45 & sc-32353 \\
\hline TRPV1 & 1 & SantaCruz & 6.06 & sc-20813 \\
\hline Piezol & 1 & SantaCruz & 3.13 & sc- 164319 \\
\hline Piezo2 & 1 & SantaCruz & 2.99 & sc- 84763 \\
\hline L-type $\mathrm{Ca}^{2+}$ & 0.5 & SantaCruz & 4.98 & sc- 25686 \\
\hline$\beta$-actin & $1,0.25,0.5,0.75,2$ & WAKO & 2.56 & 019-19741 \\
\hline
\end{tabular}

until use. Antibody microarrays were verified to remain active for at least 2 weeks after printing, and all incubations were carried out within that time frame.

Four $\times 10^{4}$ cells $/ \mathrm{mL}$ of Stro- 1 selected MSCs in $1.8 \mathrm{mg} / \mathrm{mL}$ collagen hydrogels were stimulated with $\mathrm{N} 30$ and N90. After stimulation for 1 and 2 weeks, hydrogel samples were digested with collagenase. Total protein was quantified using micro-BCA kit (Pierce, Thermo Fisher). Initially, one labeled sample was titrated $\left(2.5-15 \mu \mathrm{g} \mathrm{mL}^{-1}\right)$ for optimal signal-to-noise ratio, and all samples were subsequently incubated for 1 $\mathrm{h}$ at $23^{\circ} \mathrm{C}$ at $9 \mu \mathrm{g} \mathrm{mL}^{-1}$ in Tris-buffered saline (TBS; $20 \times 10^{-3} \mathrm{~m}$ Tris$\mathrm{HCl}, 100 \times 10^{-3} \mathrm{~m} \mathrm{NaCl}, 1 \times 10-3 \mathrm{~m} \mathrm{CaCl}_{2}, 1 \times 10^{-3} \mathrm{~m} \mathrm{MgCl} 2, \mathrm{pH}$ 7.2) with $0.05 \%$ Tween 20 (TBS-T). All microarray experiments were carried out using three replicate slides. Alexa Fluor 555 labeled MSC lysates $\left(10 \mu \mathrm{g} \mathrm{mL}^{-1}\right)$ were incubated in two separate subarrays on every slide to confirm retained antibody performance and printing, respectively. After incubation, slides were washed three times in TBS$\mathrm{T}$ for $2 \mathrm{~min}$ per wash, once in TBS, and then centrifuged dry. Dried slides were scanned immediately on an Agilent G2505 microarray scanner using the Cy3 channel (532 nm excitation, 90\% photomultiplier tubes (РMT), $5 \mu \mathrm{m}$ resolution), and intensity data were saved as.tif files. Data were normalized to the mean of three replicate microarray slides (subarray-by-subarray using subarray total intensity, $n$ $=3,18$ data points). $\beta$-actin was used as internal protein control. Heatmaps were generated by Hierarchical Clustering Explorer v3.0.

Metabolomics. Stro-1-selected MSCs seeded with $4 \times 10^{4}$ cells/ $\mathrm{mL}$ density in $1.8 \mathrm{mg} / \mathrm{mL}$ collagen hydrogels were stimulated with N30 and N90. After 1 and 2 weeks NS, the gels were homogenized on ice, and metabolites were then extracted using a chloroform/methanol/ water (1:3:1 ratio) extraction buffer. Samples were agitated on a shaker at $4{ }^{\circ} \mathrm{C}$ for $1 \mathrm{~h}$ and in turn centrifuged at $13,000 \times \mathrm{g}$ at $4{ }^{\circ} \mathrm{C}$ for $5 \mathrm{~min}$. incubation, the samples were then centrifuged and resuspended in 250 $\mu \mathrm{L}$ of flow cytometry buffer ( $2 \%$ FBS, 2 mM EDTA in $1 \times$ PBS) and transferred to 96-well plates. Resuspended MSCs in 96-well plates were incubated for $30 \mathrm{~min}$. A signal of $\mathrm{H}_{2} \mathrm{DCF}-\mathrm{DA}$ fluorescein was detected by using flow cytometry at $492-295 \mathrm{~nm}$ for excitation and 517-527 $\mathrm{nm}$ for emission.

ELISA of Interleukin-1 $\beta$. Stro-1-selected MSCs seeded with $4 \times$ $10^{4}$ cells $/ \mathrm{mL}$ density in $1.8 \mathrm{mg} / \mathrm{mL}$ collagen hydrogels were nanostimulated for 7 days. Hydrogels were digested with collagenase (Sigma-Aldrich, UK). Protein was extracted using RIPA lysis buffer containing phosphatase and protease inhibitors. Total protein concentration was quantified using BCA kits (Pierce, ThermoFisher). Human IL-1 $\beta$ kits (DY201-05, R\&D systems) were used for analysis. Working concentration of reagents (Human IL-1 $\beta$ capture antibody; 840168, Human IL-1 $\beta$ detection Antibody; 840169, Human IL-1 $\beta$ standard; 840170, Streptavidin-HRP; 893975) were prepared as per manufacturer's instructions. To coat the captured antibody onto the ELISA plate, $100 \mu \mathrm{L}$ of captured antibody was added into 96-well plate and incubated overnight at $4{ }^{\circ} \mathrm{C}$. The plate was then washed with washing buffer (0.05\% Tween 20 in $1 \times$ PBS (pH 7.4) 3 times using multichannel pipettes. $300 \mu \mathrm{L}$ of reagent diluent (Reagent diluent concentrate 2, DY995, R\&D systems) was added and incubated for $1 \mathrm{~h}$ to block the coated plate. $100 \mu \mathrm{L}$ of samples and of standards in reagent diluent (Human IL-1 $\beta, 840170$ ) were added and incubated for $2 \mathrm{~h}$ at room temperature. Samples were washed 3 times with washing buffer. $100 \mu \mathrm{L}$ of streptavidin-HRP was added and incubated for $30 \mathrm{~min}$ at room temperature. Samples were washed 3 times. $100 \mu \mathrm{L}$ of substrate solution (1:1 of color reagent $\mathrm{A} ; \mathrm{H}_{2} \mathrm{O}_{2}$ and color reagent $\mathrm{B}$; tetramethylbenzidine) were added and incubated for $20 \mathrm{~min}$. $50 \mu \mathrm{L}$ of stop solution $\left(2 \mathrm{NH}_{2} \mathrm{SO}_{4}, \mathrm{DY} 994, \mathrm{R} \& \mathrm{D}\right.$ systems $)$ was added. A 
Table 5. Details of Sample Replicates and Statistical Tests Used in Figures

\begin{tabular}{|c|c|c|c|c|c|c|}
\hline figure & sub figure & name & statistical analysis & donors & biological replicates & technical replicates \\
\hline \multirow[t]{7}{*}{1} & $\mathrm{~B}$ & Interferometry 0.8 vs $1.8 \mathrm{mg} / \mathrm{mL}$ & Mann-Whitney & - & 24 & 5 \\
\hline & $\mathrm{C}$ & Interferometry Freq-amplitude & - & - & $3-5$ & 5 \\
\hline & $\mathrm{D}$ & Interferometry Plateform-gel & Mann-Whitney & - & 24 & 5 \\
\hline & $\mathrm{E}$ & Interferometry Vol-amplitude & - & - & 5 & 5 \\
\hline & $\mathrm{F}$ & Interferometry Freq-amplitude & - & - & 5 & 5 \\
\hline & G & RT-PCR day9 & One-way ANOVA/Tukey & 2 & 4 & 3 \\
\hline & $\mathrm{H}$ & temporal gene analysis of phenotype & Mann-Whitney & 3 & 4 & 3 \\
\hline \multirow[t]{5}{*}{2} & A & protein array heatmap & - & 1 & 4 & - \\
\hline & $\mathrm{B}$ & PCR-ion channel & One-way ANOVA/Tukey & 2 & 4 & 3 \\
\hline & $\mathrm{C}$ & PCR-BMP2 & One-way ANOVA/Tukey & 2 & 4 & 3 \\
\hline & $\mathrm{D}$ & PCR-CollA & One-way ANOVA/Tukey & 2 & 4 & 3 \\
\hline & $\mathrm{E}$ & PCR-ROCK inhibition & One-way ANOVA/Tukey & 1 & 4 & 3 \\
\hline 3 & A-D & metabolomics & - & 1 & 4 & - \\
\hline \multirow[t]{3}{*}{4} & A-C, F & metabolomics & - & 1 & 4 & - \\
\hline & $\mathrm{B}$ & ROS (DCF-DA) & One-way ANOVA/Tukey & 3 & 3 & 1 \\
\hline & $\mathrm{E}$ & PCR, ROS with NAC & One-way ANOVA/Tukey & 1 & 4 & 3 \\
\hline \multirow[t]{10}{*}{5} & A & PCR inflammation & Mann-Whitney & 1 & 4 & 3 \\
\hline & $\mathrm{B}$ & PCR, Effect of dependent pathway & Mann-Whitney & 1 & 4 & 3 \\
\hline & $\mathrm{C}$ & PCR-temporal gene study & One-way ANOVA/Tukey & 3 & 4 & 3 \\
\hline & $\mathrm{D}$ & ELISA- $1 \beta$ & One-way ANOVA/Tukey & 1 & 4 & 1 \\
\hline & $\mathrm{C}$ & Contraction & Two-way ANOVA/Tukey & 1 & 4 & 1 \\
\hline & $\mathrm{D}$ & Interferometry & - & - & $3-6$ & 5 \\
\hline & $\mathrm{E}$ & Interferometry Freq-amp & - & - & 3 & 5 \\
\hline & $\mathrm{F}$ & direction & - & 1 & 1 & 10 \\
\hline & G & velocity & Mann-Whitney & 1 & 1 & 10 \\
\hline & $\mathrm{H}$ & cryosection and staining & - & 1 & 2 & - \\
\hline \multirow[t]{2}{*}{7} & A,B & PCR, temporal study & One-way ANOVA/Tukey & 2 & 4 & 3 \\
\hline & $\mathrm{D}$ & PCR-memory test & Mann-Whitney & 1 & 4 & 3 \\
\hline \multirow[t]{3}{*}{ S1 } & A & rheology & Mann-Whitney & - & 3 & 1 \\
\hline & $\mathrm{B}$ & hydrogel contraction & Two-way ANOVA/Tukey & 1 & 4 & 1 \\
\hline & $\mathrm{C}$ & AlamarBlue & One-way ANOVA/Tukey & 1 & $3-5$ & 1 \\
\hline S2 & - & PCR-non responding pt-NFkB & One-way ANOVA/Tukey & 1 & 4 & 3 \\
\hline \multirow[t]{2}{*}{ S3 } & - & & -Paired- $t$ test (same gel) & 1 & 4 & 1 \\
\hline & & & -One-way ANOVA/Tukey & & & \\
\hline
\end{tabular}

microplate reader was used to determine the optical density at 450 and $570 \mathrm{~nm}$.

Statistics. To compare the means of samples of more than two groups, one-way ANOVA with a Tukey post hoc test was used in qRTPCR and AlamarBlue assays. Two-way ANOVA with Tukey post hoc test was used to analyze hydrogel and composites contraction by times. To compare the data between two groups, two tailed, paired t-tests were used for AlamarBlue Assay of composites. Two tailed, Mann-Whitney $\mathrm{U}$ tests were used in interferometric measurement and qRT-PCR. Biological sample populations with four replicates were always used. All results are shown in mean \pm standard deviation with $95 \%, 99 \%$, and $99.9 \%$ of accuracy ( $* P \leq 0.5$, ** $P \leq 0.01$, *** $P \leq 0.001)$. Replicate details for each experiment is shown in Table 5 .

\section{ASSOCIATED CONTENT}

\section{SI Supporting Information}

The Supporting Information is available free of charge at https://pubs.acs.org/doi/10.1021/acsnano.0c03130.

Supporting Figures $1-5$ provide detail on nanovibrational bioreactor design, rheology, and gel contraction, metabolomics analysis, and testing cells from a nonresponsive donor (PDF)

\section{AUTHOR INFORMATION}

\section{Corresponding Author}

Matthew J. Dalby - Centre for the Cellular Microenvironment, Institute of Molecular, Cell and Systems Biology, College of Medical, Veterinary, and Life Sciences, University of Glasgow, Glasgow G12 8QQ, United Kingdom; 으 orcid.org/0000-00020528-3359; Email: matthew.dalby@glasgow.ac.uk

\section{Authors}

Wich Orapiriyakul - Centre for the Cellular Microenvironment, Institute of Molecular, Cell and Systems Biology, College of Medical, Veterinary, and Life Sciences, University of Glasgow, Glasgow G12 8QQ, United Kingdom; Department of Orthopedics, Faculty of Medicine, Prince of Songkla University, Songkhla 90110, Thailand

Monica P. Tsimbouri - Centre for the Cellular Microenvironment, Institute of Molecular, Cell and Systems Biology, College of Medical, Veterinary, and Life Sciences, University of Glasgow, Glasgow G12 8QQ, United Kingdom

Peter Childs - Centre for the Cellular Microenvironment, Division of Biomedical Engineering, School of Engineering, University of Glasgow, Glasgow G12 8LT, United Kingdom

Paul Campsie - SUPA Department of Biomedical Engineering, University of Strathclyde, Glasgow G1 1QE, United Kingdom 
Julia Wells - Bone and Joint Research Group, Centre for Human Development, Stem Cells and Regeneration, Institute of Developmental Sciences, University of Southampton, Southampton SO16 6YD, United Kingdom

Marc A. Fernandez-Yague - Centre for Research in Medical Devices (CÚRAM), National University of Ireland Galway, Galway, Ireland

Karl Burgess - Glasgow Polyomics, College of Medical, Veterinary, and Life Sciences, University of Glasgow, Glasgow G61 1BD, United Kingdom

K. Elizabeth Tanner - Centre for the Cellular Microenvironment, Division of Biomedical Engineering, School of Engineering, University of Glasgow, Glasgow G12 8LT, United Kingdom; School of Engineering and Materials Science, Queen Mary University of London, London E1 4NS, United Kingdom; (1) orcid.org/0000-0003-2257-0218

Manlio Tassieri - Centre for the Cellular Microenvironment, Division of Biomedical Engineering, School of Engineering, University of Glasgow, Glasgow G12 8LT, United Kingdom; (ㄱ) orcid.org/0000-0002-6807-0385

Dominic Meek - Department of Orthopedics, Queen Elizabeth II University Hospital, Glasgow G51 4TF, United Kingdom

Massimo Vassalli - Centre for the Cellular Microenvironment, Division of Biomedical Engineering, School of Engineering, University of Glasgow, Glasgow G12 8LT, United Kingdom; (1) orcid.org/0000-0002-3063-4376

Manus J. P. Biggs - Centre for Research in Medical Devices (CÚRAM), National University of Ireland Galway, Galway, Ireland

Manuel Salmeron-Sanchez - Centre for the Cellular Microenvironment, Division of Biomedical Engineering, School of Engineering, University of Glasgow, Glasgow G12 8LT, United Kingdom; (1) orcid.org/0000-0002-8112-2100

Richard O. C. Oreffo - Bone and Joint Research Group, Centre for Human Development, Stem Cells and Regeneration, Institute of Developmental Sciences, University of Southampton, Southampton SO16 6YD, United Kingdom

Stuart Reid - SUPA Department of Biomedical Engineering, University of Strathclyde, Glasgow G1 1QE, United Kingdom

Complete contact information is available at:

https://pubs.acs.org/10.1021/acsnano.0c03130

\section{Author Contributions}

W.O., P.M.T., M.J.D., K.E.T., M.J.P.B., S.R., M.S.-S., and R.O.C.O. conceived the experiments. W.O., P.M.T., K.B., M.F.Y., M.T., K.E.T., P.G.C., and P.C. performed the experiments. J.W., R.O.C.O., M.T., M.F.Y., W.O., R.M.D.M., P.C., S.R., and M.S.-S. provided materials and expertise. P.M.T., M.J.D., and P.C. analyzed the data. M.V., R.M.D.M., and R.O.C.O. provided critique and context for the data. W.O., P.M.T., and M.J.D. wrote the manuscript. W.O. and M.J.D. prepared the figures. All authors read and commented on the manuscript.

\section{Notes}

The authors declare no competing financial interest.

Table 5 raw data can be accessed at DOI: 10.5525/ gla.researchdata.1037.

\section{ACKNOWLEDGMENTS}

W.O. was supported by a scholarship from the Royal Thai Government and Faculty of medicine, Prince of Songkla University. The work was supported by grants to M.J.D. from
BBSRC (BB/P00220X/1, BB/S018808/1), EPSRC (EP/ N013905/1, EP/P001114/1) and the Sir Bobby Charlton Foundation and to M.J.P.B from Science Foundation Ireland (16/BBSRC/3317). We thank Mrs. Carol-Anne Smith for technical assistance. We thank Jane Alfred, Ph.D., from Catalyst Editorial (www.catalyst-editorial.co.uk) for editing a draft of this manuscript.

\section{REFERENCES}

(1) Giannoudis, P. V.; Chris Arts, J. J.; Schmidmaier, G.; Larsson, S. What Should Be the Characteristics of the Ideal Bone Graft Substitute? Injury 2011, 42, S1-S2.

(2) Myeroff, C.; Archdeacon, M. Autogenous Bone Graft: Donor Sites and Techniques. J. Bone Jt. Surg., Am. Vol. 2011, 93, 2227-2236.

(3) Dimitriou, R.; Mataliotakis, G. I.; Angoules, A. G.; Kanakaris, N. K.; Giannoudis, P. V. Complications following Autologous Bone Graft Harvesting from the Iliac Crest and Using the RIA: a Systematic Review. Injury 2011, 42, S3-S15.

(4) Galipeau, J.; Sensebe, L. Mesenchymal Stromal Cells: Clinical Challenges and Therapeutic Opportunities. Cell Stem Cell 2018, 22, 824-833.

(5) Bianco, P.; Cao, X.; Frenette, P. S.; Mao, J. J.; Robey, P. G.; Simmons, P. J.; Wang, C. Y. The Meaning, the Sense and the Significance: Translating the Science of Mesenchymal Stem Cells into Medicine. Nat. Med. 2013, 19, 35-42.

(6) Kanczler, J.; Tare, R. S.; Stumpf, P.; Noble, T. J.; Black, C.; Oreffo, R. O. C. Isolation, Differentiation, and Characterization of Human Bone Marrow Stem Cells In Vitro and In Vivo. Methods Mol. Biol. 2019, 1914, 53-70.

(7) Tare, R. S.; Babister, J. C.; Kanczler, J.; Oreffo, R. O. Skeletal Stem Cells: Phenotype, Biology and Environmental Niches Informing Tissue Regeneration. Mol. Cell. Endocrinol. 2008, 288, 11-21.

(8) Nikukar, H.; Reid, S.; Tsimbouri, P. M.; Riehle, M. O.; Curtis, A. S.; Dalby, M. J. Osteogenesis of Mesenchymal Stem Cells by Nanoscale Mechanotransduction. ACS Nano 2013, 7, 2758-2767.

(9) Tsimbouri, P. M.; Childs, P. G.; Pemberton, G. D.; Yang, J.; Jayawara, V.; Oripiriyakul, W.; Burgess, K. V.; Gonzales-Garcia, C.; Blackburn, G.; Thomas, D.; Vallejo-Giraldo, C.; Biggs, M. J. P.; Curtis, A. S. G.; Salmeron-Sanchez, M.; Reid, S.; Dalby, M. J. Stimulation of 3D Osteogenesis by Mesenchymal Stem Cells Using a Nanovibrational Bioreactor. Nature Biomedical Engineering 2017, 1, 758-770.

(10) Campsie, P.; Childs, P. G.; Robertson, S. N.; Cameron, K.; Hough, J.; Salmeron-Sanchez, M.; Tsimbouri, P. M.; Vichare, P.; Dalby, M. J.; Reid, S. Design, Construction and Characterisation of a Novel Nanovibrational Bioreactor and Cultureware for Osteogenesis. Sci. Rep. 2019, 9, 12944.

(11) Wen, J. H.; Vincent, L. G.; Fuhrmann, A.; Choi, Y. S.; Hribar, K. C.; Taylor-Weiner, H.; Chen, S.; Engler, A. J. Interplay of Matrix Stiffness and Protein Tethering in Stem Cell Differentiation. Nat. Mater. 2014, 13, 979.

(12) Engler, A. J.; Sen, S.; Sweeney, H. L.; Discher, D. E. Matrix Elasticity Directs Stem Cell Lineage Specification. Cell 2006, 126, 677689.

(13) Kell, G. S.; Whalley, E. The PVT Properties of Water. Philos. Trans. R. Soc., A 1965, 258, 565-614.

(14) Stein, G. S.; Lian, J. B. Molecular Mechanisms Mediating Proliferation/Differentiation Interrelationships during Progressive Development of the Osteoblast Phenotype. Endocr. Rev. 1993, 14, 424-442.

(15) Yang, J.; McNamara, L. E.; Gadegaard, N.; Alakpa, E. V.; Burgess, K. V.; Meek, R. M.; Dalby, M. J. Nanotopographical Induction of Osteogenesis through Adhesion, Bone Morphogenic Protein Cosignaling, and Regulation of MicroRNAs. ACS Nano 2014, 8, 9941-9953.

(16) Loeser, R. F. Chondrocyte Integrin Expression and Function. Biorheology 2000, 37, 109-116.

(17) Clapham, D. E. TRP Channels as Cellular Sensors. Nature 2003, 426, 517-524. 
(18) Vandorpe, D. H.; Morris, C. E. Stretch Activation of the Aplysia S-Channel. J. Membr. Biol. 1992, 127, 205-214.

(19) Masuyama, R.; Vriens, J.; Voets, T.; Karashima, Y.; Owsianik, G.; Vennekens, R.; Lieben, L.; Torrekens, S.; Moermans, K.; Vanden Bosch, A.; Bouillon, R.; Nilius, B.; Carmeliet, G. TRPV4-Mediated Calcium Influx Regulates Terminal Differentiation of Osteoclasts. Cell Metab. 2008, 8, 257-265.

(20) Lee, W.; Leddy, H. A.; Chen, Y.; Lee, S. H.; Zelenski, N. A.; McNulty, A. L.; Wu, J.; Beicker, K. N.; Coles, J.; Zauscher, S.; Grandl, J.; Sachs, F.; Guilak, F.; Liedtke, W. B. Synergy Between Piezo1 And Piezo2 Channels Confers High-Strain Mechanosensitivity to Articular Cartilage. Proc. Natl. Acad. Sci. U. S. A. 2014, 111, E5114-5122.

(21) Zhang, W.; Yan, Z.; Jan, L. Y.; Jan, Y. N. Sound Response Mediated by the TRP Channels NOMPC, NANCHUNG, and INACTIVE in Chordotonal Organs of Drosophila Larvae. Proc. Natl. Acad. Sci. U. S. A. 2013, 110, 13612-13617.

(22) Maksimovic, S.; Nakatani, M.; Baba, Y.; Nelson, A. M.; Marshall, K. L.; Wellnitz, S. A.; Firozi, P.; Woo, S. H.; Ranade, S.; Patapoutian, A.; Lumpkin, E. A. Epidermal Merkel Cells Are Mechanosensory Cells that Tune Mammalian Touch Receptors. Nature 2014, 509, 617-621.

(23) Henstock, J. R.; Rotherham, M.; El Haj, A. J. Magnetic Ion Channel Activation of TREK1 in Human Mesenchymal Stem Cells Using Nanoparticles Promotes Osteogenesis in Surrounding Cells. J. Tissue Eng. 2018, 9, 2041731418808695.

(24) Nourse, J. L.; Pathak, M. M. How Cells Channel Their Stress: Interplay between Piezol and the Cytoskeleton. Semin. Cell Dev. Biol. 2017, 71, 3-12.

(25) Wei, L.; Mousawi, F.; Li, D.; Roger, S.; Li, J.; Yang, X.; Jiang, L. H. Adenosine Triphosphate Release and P2 Receptor Signaling in Piezol Channel-Dependent Mechanoregulation. Front. Pharmacol. 2019, 10, 1304.

(26) Creek, D. J.; Jankevics, A.; Breitling, R.; Watson, D. G.; Barrett, M. P.; Burgess, K. V. Towards Global Metabolomics Analysis with Hydrophilic Interaction Liquid Chromotagography Mass Spectrometry: Improved Metabolite Identification by Retention Time Prediction. Anal. Chem. 2011, 83, 8703-8710.

(27) Hamanaka, R. B.; Chandel, N. S. Warburg Effect and Redox Balance. Science 2011, 334, 1219-1220.

(28) Alberts, B.; Bray, D.; Lewis, J.; Raff, M.; Roberts, K.; Watson, J. D. How Cells Obtain Energy from Food. In Mol. Biol. Cell, 4th ed.; Gartland Science: New York, 2002.

(29) Byon, C. H.; Javed, A.; Dai, Q.; Kappes, J. C.; Clemens, T. L.; Darley-Usmar, V. M.; McDonald, J. M.; Chen, Y. Oxidative Stress Induces Vascular Calcification through Modulation of the Osteogenic Transcription Factor Runx2 by AKT Signaling. J. Biol. Chem. 2008, 283, 15319-15327.

(30) Lin, C. H.; Li, N. T.; Cheng, H. S.; Yen, M. L. Oxidative Stress Induces Imbalance of Adipogenic/Osteoblastic Lineage Commitment in Mesenchymal Stem Cells through Decreasing SIRT1 Functions. J. Cell. Mol. Med. 2017, 22, 786-796.

(31) Atashi, F.; Modarressi, A.; Pepper, M. S. The Role of Reactive Oxygen Species in Mesenchymal Stem Cell Adipogenic and Osteogenic Differentiation: A Review. Stem Cells Dev. 2015, 24, 1150-1163.

(32) Kilian, K. A.; Bugarija, B.; Lahn, B. T.; Mrksich, M. Geometric Cues for Directing the Differentiation of Mesenchymal Stem Cells. Proc. Natl. Acad. Sci. U. S. A. 2010, 107, 4872-4877.

(33) Dalby, M. J.; Garcia, A. J.; Salmeron-Sanchez, M. Receptor Control in Mesenchymal Stem Cell Engineering. Nat. Rev. Mater. 2018, 3, 17091 .

(34) Ge, C.; Xiao, G.; Jiang, D.; Franceschi, R. T. Critical Role of the Extracellular Signal-Regulated Kinase-MAPK Pathway in Osteoblast Differentiation and Skeletal Development. J. Cell Biol. 2007, 176, 709718.

(35) Birben, E.; Sahiner, U. M.; Sackesen, C.; Erzurum, S.; Kalayci, O. Oxidative Stress and Antioxidant Defense. World Allergy Organ. J. 2012, 5, 9-19.

(36) Chen, C. T.; Shih, Y. R.; Kuo, T. K.; Lee, O. K.; Wei, Y. H. Coordinated Changes of Mitochondrial Biogenesis and Antioxidant
Enzymes during Osteogenic Differentiation of Human Mesenchymal Stem Cells. Stem Cells 2008, 26, 960-968.

(37) Wang, L.; Zhao, X.; Wei, B. Y.; Liu, Y.; Ma, X. Y.; Wang, J.; Cao, P. C.; Zhang, Y.; Yan, Y. B.; Lei, W.; Feng, Y. F. Insulin Improves Osteogenesis of Titanium Implants under Diabetic Conditions by Inhibiting Reactive Oxygen Species Overproduction Via the PI3K-Akt Pathway. Biochimie 2015, 108, 85-93.

(38) Mills, E. L.; Kelly, B.; Logan, A.; Costa, A. S. H.; Varma, M.; Bryant, C. E.; Tourlomousis, P.; Dabritz, J. H. M.; Gottlieb, E.; Latorre, I.; Corr, S. C.; McManus, G.; Ryan, D.; Jacobs, H. T.; Szibor, M.; Xavier, R. J.; Braun, T.; Frezza, C.; Murphy, M. P.; O’Neill, L. A. Succinate Dehydrogenase Supports Metabolic Repurposing of Mitochondria to Drive Inflammatory Macrophages. Cell 2016, 167, 457-470.

(39) Loi, F.; Cordova, L. A.; Pajarinen, J.; Lin, T. H.; Yao, Z.; Goodman, S. B. Inflammation, Fracture and Bone Repair. Bone 2016, $86,119-130$.

(40) Nakano, H.; Nakajima, A.; Sakon-Komazawa, S.; Piao, J. H.; Xue, X.; Okumura, K. Reactive Oxygen Species Mediate Crosstalk between NF-Kappab and JNK. Cell Death Differ. 2006, 13, 730-737.

(41) Coulthard, L. R.; White, D. E.; Jones, D. L.; McDermott, M. F.; Burchill, S. A. P38(MAPK): Stress Responses from Molecular Mechanisms to Therapeutics. Trends Mol. Med. 2009, 15, 369-379.

(42) Tanaka, T.; Narazaki, M.; Kishimoto, T. IL-6 in Inflammation, Immunity, and Disease. Cold Spring Harbor Perspect. Biol. 2014, 6, a016295.

(43) Cassatella, M. A.; Meda, L.; Bonora, S.; Ceska, M.; Constantin, G. Interleukin 10 (IL-10) Inhibits the Release of Proinflammatory Cytokines from Human Polymorphonuclear Leukocytes. Evidence for an Autocrine Role of Tumor Necrosis Factor and IL-1 Beta in Mediating the Production of IL-8 Triggered by Lipopolysaccharide. J. Exp. Med. 1993, 178, 2207-2211.

(44) Hou, J.; Sun, Y.; Chen, F.; Yu, L.; Mao, Q.; Wang, L.; Guo, X.; Liu, C. Analysis of Microstructures and Macrotextures for Different Apple Cultivars Based on Parenchyma Morphology. Microsc. Res. Tech. 2016, 79, 304-312.

(45) Cortright, D. N.; Krause, J. E.; Broom, D. C. TRP Channels and Pain. Biochim. Biophys. Acta, Mol. Basis Dis. 2007, 1772, 978-988.

(46) Montell, C. The TRP Superfamily of Cation Channels. Sci. Signaling 2005, 2005 (272), re3.

(47) Elosegui-Artola, A.; Andreu, I.; Beedle, A. E. M.; Lezamiz, A.; Uroz, M.; Kosmalska, A. J.; Oria, R.; Kechagia, J. Z.; Rico-Lastres, P.; Le Roux, A. L.; Shanahan, C. M.; Trepat, X.; Navajas, D.; Garcia-Manyes, S.; Roca-Cusachs, P. Force Triggers YAP Nuclear Entry by Regulating Transport across Nuclear Pores. Cell 2017, 171, 1397-1410.

(48) Yang, C.; Tibbitt, M. W.; Basta, L.; Anseth, K. S. Mechanical Memory and Dosing Influence Stem Cell Fate. Nat. Mater. 2014, 13, $645-652$

(49) Miyamoto, T.; Mochizuki, T.; Nakagomi, H.; Kira, S.; Watanabe, M.; Takayama, Y.; Suzuki, Y.; Koizumi, S.; Takeda, M.; Tominaga, M. Functional Role for Piezol in Stretch-Evoked $\mathrm{Ca}(2)(+)$ Influx and ATP Release in Urothelial Cell Cultures. J. Biol. Chem. 2014, 289, 16565-16575.

(50) Sugimoto, A.; Miyazaki, A.; Kawarabayashi, K.; Shono, M.; Akazawa, Y.; Hasegawa, T.; Ueda-Yamaguchi, K.; Kitamura, T.; Yoshizaki, K.; Fukumoto, S.; Iwamoto, T. Piezo Type Mechanosensitive Ion Channel Component 1 Functions as a Regulator of the Cell Fate Determination of Mesenchymal Stem Cells. Sci. Rep. 2017, 7, 17696.

(51) Lewis, A. H.; Cui, A. F.; McDonald, M. F.; Grandl, J. Transduction of Repetitive Mechanical Stimuli by Piezo1 and Piezo2 Ion Channels. Cell Rep. 2017, 19, 2572-2585.

(52) Jin, P.; Jan, L. Y.; Jan, Y. N. Mechanosensitive Ion Channels: Structural Features Relevant to Mechanotransduction Mechanisms. Annu. Rev. Neurosci. 2020, 43, 207.

(53) Pattappa, G.; Heywood, H. K.; de Bruijn, J. D.; Lee, D. A. The Metabolism of Human Mesenchymal Stem Cells during Proliferation and Differentiation. J. Cell. Physiol. 2011, 226, 2562-2570.

(54) Klontzas, M. E.; Vernardis, S. I.; Heliotis, M.; Tsiridis, E.; Mantalaris, A. Metabolomics Analysis of the Osteogenic Differentiation 
of Umbilical Cord Blood Mesenchymal Stem Cells Reveals Differential Sensitivity to Osteogenic Agents. Stem Cells Dev. 2017, 26, 723-733.

(55) Murphy, M. P. How Mitochondria Produce Reactive Oxygen Species. Biochem. J. 2009, 417, 1-13.

(56) Tsimbouri, P. M.; Childs, P. G.; Pemberton, G. D.; Yang, J.; Jayawarna, V.; Orapiriyakul, W.; Burgess, K.; Gonzalez-Garcia, C.; Blackburn, G.; Thomas, D.; Vallejo-Giraldo, C.; Biggs, M. J. P; Curtis, A. S. G.; Salmeron-Sanchez, M.; Reid, S.; Dalby, M. J. Stimulation of 3D osteogenesis by mesenchymal stem cells using a nanovibrational bioreactor. Nat. Biomed. Engin. 2017, 1, 758-770.

(57) Livak, K. J.; Schmittgen, T. D. Analysis of Relative Gene Expression Data Using Real-Time Quantitative PCR and the 2(-Delta Delta C(T)) Method. Methods 2001, 25, 402-408. 